OAK RIDGE NATIONAL LABORATORY

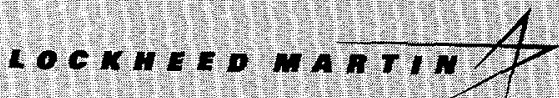

MANAGED AND OPERATED BY

LOCKHEED MARTN ENERGY RESEARCH CORPORATION FOA THE UNTEO STATES

DEPARTUENT OF ENERGY

RECEIVEO

OCT 021998

OSTI

\section{Analysis of Neutron Scattering Data: Visualization and Parameter Estimation}

\author{
J. J. Beauchamp \\ V. Fedorov \\ W. A. Hamilton \\ M. Yethiraj
}


This report has been reproduced directly from the best available copy.

Available to DOE and DOE contractors from the Office of Scientific and Technical Information, P.O. Box 62, Oak Ridge, TN 37831; prices available from (615) 576-8401.

Available to the public from the National Technical Information Service, U.S. Department of Commerce, 5285 Port Royal Rd., Springfield, VA 22161.

This report was prepared as an account of work sponsored by an agency of the United States Government. Neither the United States nor any agency thereof, nor any of their employees, makes any warranty, express or implied, or assumes any legal liability or responsibility for the accuracy, completeness, or usefulness of any information, apparatus, product, or process disclosed, or represents that its use would not infringe privately owned rights. Reference herein to any specific commercial product, process, or service by trade name, trademark, manufacturer, or otherwise, does not necessarily constitute or imply its endorsement, recommendation, or favoring by the United States Government or any agency thereof. The views and opinions of authors expressed herein do not necessarily state or reflect those of the United States Government or any agency thereof. 


\section{DISCLAIMER}

Portions of this document may be illegible in electronic image products. Images are produced from the best available original document. 


\section{ANALYSIS OF NEUTRON SCATTERING DATA: VISUALIZATION AND PARAMETER ESTIMATION}

J. J. Beauchamp and V. Fedorov

Computer Science and Mathematics Division

W. A. Hamilton and M. Yethiraj

Solid State Division

Date Published: September 1998

Prepared by

OAK RIDGE NATIONAL LABORATORY

Oak Ridge, Tennessee 37831-6285

Managed by

LOCKHEED MARTIN ENERGY RESEARCH CORP.

for the

U.S. DEPARTMENT OF ENERGY

under contract DE-AC05-96OR22464 
$\cdot$ 


\section{Preface}

This paper represents collaboration between neutron scattering experimentalists and statisticians and addresses the analysis of the massive and noisy data sets generated by small-angle neutron scattering experiments, particularly those using large arrays of detectors. The statistical techniques are based on the iterated maximum likelihood estimators. Whenever it is possible, various physically sound models are introduced and statistically compared to get the most accurate and stable estimates of the parameters that are of interest to a practitioner.

The main results included in the paper have been completed in the framework of the Oak Ridge National Laboratory Director Research and Development Fund Project "Visualization and Analysis of Data from Novel Neutron Scattering Experiments." We thank Darryl Downing who participated in the earlier stages of the research, Chuck Bayne and George Ostrouchov for reading the text and making valuable comments. 
, 


\section{Contents}

1 INTRODUCTION 1

2 TRADITIONAL APPROACHES IN DATA ANALYSIS FOR SANS 1

3 PARAMETER ESTIMATION $\quad 5$

3.1 Data . . . . . . . . . . . . . . . . . . . . . . 5

3.2 Sensitivity Analysis . . . . . . . . . . . . . . . . . 5

3.3 Fitting the Compound Model . . . . . . . . . . . . . . . 8

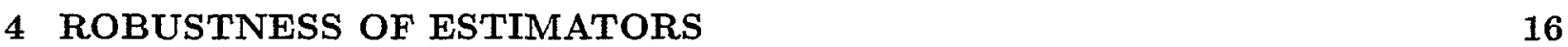

5 EXPERIMENTAL DESIGN $\quad 20$

$\begin{array}{lr}\text { APPENDIX } & 26\end{array}$

$\begin{array}{lr}\text { REFERENCES } & 26\end{array}$ 



\section{List of Figures}

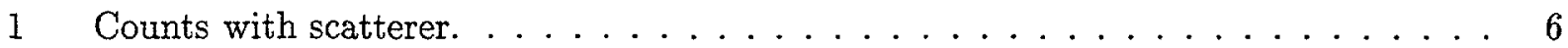

2 Counts with scatterer removed. . . . . . . . . . . . . . . . 6

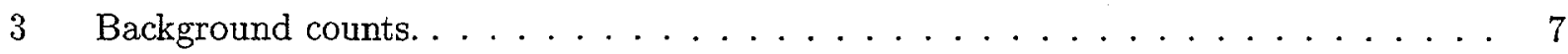

4 Scattering counts on water target. $\ldots \ldots \ldots \ldots \ldots \ldots \ldots \ldots \ldots$

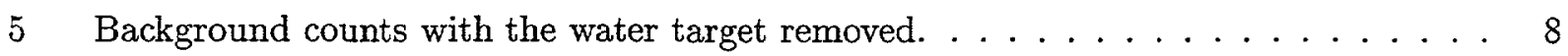

6 Estimated sensitivity function $\hat{\psi}(x, y) \ldots \ldots \ldots \ldots \ldots$

7 Residual vs momentum for the Guinier model. . . . . . . . . . . . . . 11

8 Residual vs momentum for the Rayleigh model. . . . . . . . . . . . . . . . . 11

9 Residual vs momentum for the two-exponential model. . . . . . . . . . . . . 12

10 Data and fitted model $(17)$ for $\eta_{1}\left(q^{2}\right) \ldots \ldots \ldots \ldots$

11 Obsered vs predicted intensity for model $(17)$ as $\eta_{1}\left(q^{2}\right) \ldots \ldots \ldots 14$

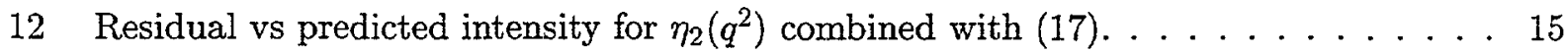

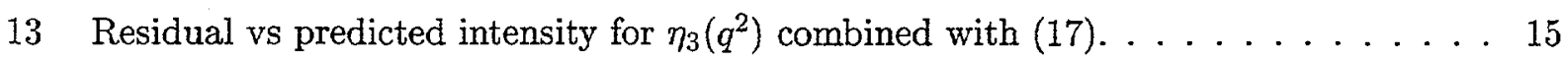

14 Data and fitted model (17) for $\eta_{1}\left(q^{2}\right)$ with $\theta_{12}=2 \theta_{14}=136.0 \mathrm{~nm} . \ldots \ldots 16$

15 The standard error of $\hat{\theta}_{12}$ as a function of $t_{1} \ldots \ldots \ldots \ldots \ldots$ 


\section{List of Tables}

$1 \quad$ Parameter estimates . . . . . . . . . . . . . . . . . . 13

2 Parameter estimates from robustness study $\ldots \ldots \ldots \ldots \ldots$

3 Parameter estimates for reduced model . . . . . . . . . . . . . . 18

4 Estimates of $\theta_{12}$ for $\gamma=0.95,0.005<q^{2}<q_{\max }^{2} \ldots \ldots \ldots \ldots$

$5 \quad$ Rayleigh-type models for $\gamma=0.95,0.005<q^{2}<q_{\max }^{2} \ldots \ldots \ldots$ 

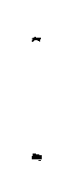


\section{INTRODUCTION}

Traditionally, small-angle neutron and x-ray scattering (SANS and SAXS) data analysis requires measurements of the signal and corrections due to the empty sample container, detector efficiency and time-dependent background. These corrections are then made on a pixel-by-pixel basis and estimates of relevant parameters (e.g., the radius of giration) are made using the corrected data. This study was carried out in order to determine whether treatment of the detector efficiency and empty sample cell in a more statistically sound way would significantly reduce the uncertainties in the parameter estimators. Elements of experiment design are shortly discussed in this paper. For instance, we studied the way the time for a measurement should be optimally divided between the counting for signal, background and detector efficiency.

In Section 2 we introduce the commonly accepted models for small-angle neutron and $\mathrm{x}$-scattering and confine ourselves to the Guinier and Rayleigh models and their minor generalizations. The traditional approaches of data analysis are discussed only to the extent necessary to allow their comparison with the proposed techniques. Section 3 describes the main stages of the proposed method: visual data exploration, fitting the detector sensitivity function, and fitting a compound model. This model includes three additive terms describing scattering by the sampler, scattering with an empty container and a background noise. We compare a few alternatives for the first term by applying various scatter plots and computing sums of standardized squared residuals. Possible corrections due to smearing effects and randomness of estimated parameters are also shortly discussed. In Section 4 the robustness of the estimators with respect to low and upper bounds imposed on the momentum value is discussed. We show that for the available data set the most accurate and stable estimates are generated by models containing double terms either of Guinier's or Rayleigh's type. The optimal partitioning of the total experimantal time between measuring various signals is discussed in Section 5 . We applied a straightforward optimization instead of some special experimental techniques because of the numerical simplicity of the corresponding problem. As a criterion of optimality we selected the variance of the giration radius maximum likelihood estimator.

The statistical background of the proposed approach is given in the appendix. The properties of the maximum likelihood estimators and the corresponding iterated estimator together with its possible numerical realization are presented in subsection A.1. In subsection A.2 we prove that the use of a compound model leads to more efficient estimators than a stage-wise analysis of different components entering that model.

\section{TRADITIONAL APPROACHES IN DATA ANALYSIS FOR SANS}

The techniques of small angle neutron and $\mathrm{x}$-ray scattering, as practiced at the ORNL $30 \mathrm{~m}$ SANS and $10 \mathrm{~m}$ SAXS facilities, are concerned with the measurement of material structures on size ranges between 1 and $1000 \mathrm{~nm}$. This size range begins just above the length scales where the positions of individual atoms must be considered (the province of crystallography). Near the lower end of this scale are aggregates of a few tens to thousands of atoms, for instance, micelles in solution, proteins, and precipitate (or void) regions in alloys. At the higher end are viruses and colloidally suspended particles in paints, while features in the organization of extended macromolecules, like polymers, cover almost the entire range [Windsor (1988)]. 
In any diffraction technique, such as small angle scattering, the relationship between the length scale or periodicity, $d$, probed when radiation of wavelength, $\lambda$, is scattered through an angle $2 \theta$ is given by the Bragg relationship familiar from crystallography

$$
\lambda=2 d \sin \theta \text {. }
$$

Since the periodicity in a sample has a direction as well as a magnitude, we use a vector to describe the scattering. The magnitude of this scattering vector, $\mathbf{q}$, is

$$
q=4 \pi(\sin \theta) / \lambda
$$

with the length scale related as $q=2 \pi / d$. The general scattering pattern from a nonperiodic sample of the sort occurring in most small angle experiments may be thought of as the superposition of reflections from all periodicities present - as in a Fourier transform. In fact, the macroscopic scattering cross-section $d \Sigma / d \Omega$ (i.e., total cross-section averaged over positions $\mathrm{r}$ within the sample volume $V$ ) measured in a small angle scattering experiment is given by

$$
d \Sigma / d \Omega=\frac{1}{V}\left|\int_{V} \beta(\mathbf{r}) \exp (i \mathbf{q r}) d \mathbf{r}\right|^{2}
$$

where $\beta$ denotes the local average of the scattering-length density of the sample given by the local average of the scattering from each nuclear species present, and

$$
\beta=\sum_{i} n_{i} b_{i}
$$

where $b_{i}$ is the scattering length of the species, and $n_{i}$ is its local number density, related to its local mass density and atomic weight as $n_{i}=N_{A} \rho_{i} / A_{i},\left(N_{A}\right.$ denotes Avogadro's number $)$. For small angle $x$-ray scattering, the scattering length for an atomic species is simply the product of the atomic number, $Z$, of the atom and the classical radius, $r_{e}$, of the electron, i.e., $b_{i}=Z r_{e}$ $\left(r_{e}=2.7 \mathrm{fm}\right)$. For neutrons, the scattering for nonmagnetic species is nuclear, has a magnitude of a few fermi, and varies erratically across the periodic table and also between different isotopes of the same atom. These variations constitute the major advantage of scattering neutrons rather than more intense $x$-ray beams. The neutron contrast for light atoms is typically greater than that produced by the monatomic x-ray relationship, while isotopic substitution can be used to highlight the contrast even further without altering the chemical behavior of the sample. The most widely exploited use of the latter advantage has been the substitution of deuterium $\left(b_{D}=6.7 \mathrm{fm}\right)$ for hydrogen $\left(b_{H}=-3.7 \mathrm{fm}\right)$ in polymeric and biological molecular systems.

In this report, we reexamine the analysis of small angle scattering camera data taken to improve both its statistical reliability and efficiency. A better understanding of the statistical properties of the small angle techniques should also lead to improved efficiency in data collection. To do this, we consider a measurement data set taken on the ORNL $30 \mathrm{~m}$ SANS for one of the instrument's secondary standards, i.e., well-characterized samples against which the scattering of unknown samples that are brought to the facility may be calibrated. The sample used in this investigation is an aluminum single crystal irradiated at very high neutron flux near the core of the ORNL High Flux Isotope Reactor (HFIR) reactor. To do a good approximation, the radiation damage produced by this process consists of spherical voids of nearly the same radius randomly distributed within the $11.4 \mathrm{~mm}$ thick specimen. For this sample, essentially a simple two-phase system of particles in a matrix, the scattering cross-section mentioned previously may be reduced to the form

$$
d \Sigma / d \Omega=\left(\beta_{p}-\beta_{A l}\right)^{2}(1 / V) n_{p} V_{p}^{2}|F(\mathbf{q})|^{2},
$$


indicating that the scattering cross-section is proportional to $\left(\beta_{p}-\beta_{A l}\right)^{2}$, the neutron contrast (of course, in the case of voids $\left.\beta_{p}=0\right) ; n_{p}$, the particle number density; $V_{p}^{2}$, the square of the particle volume; and a structure function $|F(\mathbf{q})|^{2}$. The term $F(\mathbf{q})$ can be viewed as the normalized Fourier transform of the scattering particle's shape and is known as the single-particle structure factor

$$
F(\mathbf{q})=\frac{1}{V_{p}} \int_{V_{p}} \exp (i \mathrm{q} r) d \mathbf{r}
$$

Although in general $F(\mathbf{q})$ is a complicated function, it may in most SANS experiments be averaged over all directions (i.e., particle orientations), so the spherical average of exp(iqr) is simply $(\sin q r) / q r$. If $V_{p}$ is a sphere of radius $R$, then from Windsor (1988)

$$
F(q)=\frac{3(\sin q R-q R \cos q R)}{(q R)^{3}}
$$

Asymptotically $(q R \longrightarrow 0)$, the previous $F(q)$ coincides with

$$
F(q)=\exp \left(-q^{2} R_{g}^{2} / 6\right)
$$

where $R_{g}{ }^{2}=3 R^{2} / 5$ and $R_{g}$ is the particle's radius of gyration. Unlike (7), the latter expression is valid for particles of any shape, if $q^{2} R_{g}{ }^{2}$ is sufficiently small. So at low $q$, the logarithm of the macroscopic scattering cross-section from any random particulate system, like our $A l$ target, standardly plotted against $q^{2}$ (a Guinier plot) should give a straight line. The slope of this line gives the measure mean particulate size and its intercept at $q=0$ the quantity $\left(\beta_{p}-\beta_{A l}\right)^{2}(1 / V) n_{p} V_{p}^{2}$. In the appropriate $q$ regime, a comparison with measurements of the $A l$ void standard may therefore be used to calibrate the size ranges and scattering strengths of unknown samples.

In its current arrangement, the ORNL $30 \mathrm{~m}$ SANS camera [Koehler (1986)] consists of the following, in series:

- a graphite monochromator system placed on a neutron beam tube which supplies a beam of neutrons of $0.475 \mathrm{~nm}$ mean wavelength with a wavelength coefficient of variation $\Delta \lambda / \lambda$ of $4 \%$

- a low-efficiency (high transmission) neutron proportional counter to monitor the incident neutron flux $\left(I_{0}\right)$;

- a collimation system consisting of two (usually) circular apertures of 0.5 to $3.5 \mathrm{~cm}$ radius separated by $7.6 \mathrm{~m}$;

- a sample position just beyond the second collimation aperture; beyond this the neutrons scattered by the sample are collected on a 2-dimensional $64 \mathrm{~cm} \times 64 \mathrm{~cm}$ position sensitive detector with a pixel resolution of $\sim 1 \mathrm{~cm}$, which can be moved in its vacuum flight tube from 1.2 to $19 \mathrm{~m}$ from the sample;

- at the detector, the unscattered incident beam is intercepted by a central beam stop. In various configurations, the instrument can measure a $q$ range spanning 0.05 to $3 \mathrm{~nm}^{-1}$.

A traditional evaluation [Dubner et al (1990); Chen and Lin (1987)] of the macroscopic SANS crosssection for a sample requires the measurement and correction for contributions to scattered intensity from the following instrument components or a sample container: the so-called empty container signal $I_{e}$; the ambient background, $I_{b}$, present during the measurement from external sources when 
a sheet of cadmium ( $C d$, a strong neutron absorber) is blocking the beam; and a correction for the efficiency of the SANS camera detector, which is itself evaluated by measurement of a scattering sample. Also required is the evaluation of the transmission of the sample (and, in most cases, the empty sample container) to correct for attenuation, caused by either absorption or scattering, of the incident neutron beam in traversing the sample. Correction for these effects to obtain the "bare" sample scattering, the measured count at each pixel (denoted by the subscript i), $I_{s, i}^{0}$ ) is done by combination of the sample, empty cell, and $C d$ background measurements normalized to incident neutron flux. Denoting the monitor counts for these runs as $M_{s}, M_{e}$, and $M_{b}$, respectively, one computes the, so-called, corrected intensity as

$$
I_{s i}^{0}=\left[\frac{I_{s i}}{M_{s}}-\frac{I_{b i}}{M_{b}}\right]-\frac{T_{s}}{T_{e}}\left[\frac{I_{e i}}{M_{e}}-\frac{I_{b i}}{M_{b}}\right]
$$

for the sample. The factor $T_{s} / T_{e}$ corrects for the attenuation difference between the sample in its container and the container's transmission (in the present case $T_{e}=100 \%$ since the Al standard is self-supporting). Let $\operatorname{Var}(X)$ stand for the expectation $E\left[\left(X-X_{0}\right)^{2}\right]$, where $X_{0}=E(X)$. Then assuming independence of all three measurements, we can derive that

$$
\operatorname{Var}\left(I_{s i}^{0}\right)=\frac{1}{M_{s}^{2}} \operatorname{Var}\left(I_{s i}\right)+\left(\frac{T_{s}}{T_{e} M_{e}}\right)^{2} \operatorname{Var}\left(I_{e i}\right)+\frac{1}{M_{b}^{2}}\left(1-\frac{T_{s}}{T_{e}}\right)^{2} \operatorname{Var}\left(I_{b i}\right)>\frac{1}{M_{s}^{2}} \operatorname{Var}\left(I_{s i}\right) .
$$

Thus, reducing the systematic bias correction (9) leads to an increase of variance of the data to be processed. The increase in the variance motivated us to develop an approach that is based on building a model comprising all three experimental stages and allowing us to estimate all the required parameters without intuitive adjustments, such as those given above. Assuming scattering cross-sections low enough (or samples thin enough) that multiple scattering is not significant, the relationship between the macroscopic cross-section of the sample at the scattering vector at pixel $i, d \Sigma_{s}\left(\mathbf{q}_{i}\right) / d \Omega$, and the corrected count is

$$
I_{s i}^{0}=\Phi_{M}\left[\frac{T_{s}}{T_{e}}\right] t_{s}\left[\frac{d \Sigma_{s}\left(\mathbf{q}_{i}\right)}{d \Omega}\right] \Delta \Omega_{i} \psi_{i}
$$

where $\Phi_{M}$ is the number of incident neutrons corresponding to a unit monitor count, $t_{s}$ is the sample thickness, $\Delta \Omega_{i}$ is the solid angle subtended by pixel $i$, and $\psi_{i}$ is the efficiency of the detector at pixel $i$, that generally varies from pixel to pixel because of the characteristics of the detector electronics.

The full measurement of the cross-section of the sample of interest, therefore, requires the calibration of the instrument characteristics contained in the product $\Phi_{M} \Delta \Omega_{i} \psi_{i}$. The $\Delta \Omega_{i}$ contribution is relatively simple, requiring only the position calibration of the detector pixel positions from a hole plate measurement in which a known pattern of holes in a $C d$ plate at the detector allows neutron scattered at the sample position to illuminate only to calculable positions on the detector.

The evaluation of the instrument factor $\Phi_{M} \psi_{i}$ requires measurement of a standard $1 \mathrm{~mm}$-thick light water sample. Due to the strong incoherent scattering of hydrogen, light water is overwhelmingly an isotropic scatterer, so the differential cross-section is constant: $d \Sigma_{H_{2} \mathrm{O}} / d \Omega=\Sigma_{\mathrm{H}_{2} \mathrm{O}} / 4 \pi$. This sample placed at the sample position scatters neutrons evenly across the detector so any apparent variations in illumination from position to position are due to variations in the detector sensitivity at that pixel $\psi_{i}$. After correction for the quartz cell holding the water (as per any liquid sample by equation (9)) and with some slight multiple scattering corrections (light water is a very strong scatterer), we may substitute the known differential cross-section for this sample into equation (10) to obtain $\Phi_{M} \psi_{i}$ at each pixel on the detector.

In the remainder of this report, we reconsider the data reduction method used to obtain the scattering cross-section for a sample of data from neutron scattering experiments. 


\section{PARAMETER ESTIMATION}

\subsection{Data}

The data used in this analysis came from the following experimental runs:

- counts $\left(I_{a}\right)$ for the aluminum target, the corresponding data set labeled by $A L 4$;

- counts $\left(I_{e}\right)$ from a run with the scatterer removed, the corresponding data set labeled by $M T 1$

- counts $\left(I_{b}\right)$ from a run when the beam is blocked with cadmium (i.e., the beam-closed background), the corresponding data set labeled by $C D 1$;

- counts $\left(I_{w}\right)$ from a raw $\mathrm{H}_{2} \mathrm{O}$ scattering run, the corresponding data set labeled by $S E N S$; and

- counts $\left(I_{w e}\right)$ from a run associated with the raw $\mathrm{H}_{2} \mathrm{O}$ scattering data when the $\mathrm{H}_{2} \mathrm{O}$ scatterer is removed, the corresponding data set labeled by $M T 2$.

Figures 1-5 provide a graphical summary of the observed counts. In all these and subsequent figures, the $x$ and $y$ axes are converted to the momentum units corresponding to the scattering on the aluminum target. The estimation of the parameters in all models discussed in this study involves a multi-stage procedure with the following stages:

- estimating the parameters in the sensitivity function $\psi$, using a polynomial approximation;

- scaling of the observed intensities in the $A L 4$ data set by dividing each observed intensity by the product of the target transparency factor $\gamma$ and the estimated sensitivity from the first stage;

- scaling of the observed intensities in the $M T 1$ and $C D 1$ data sets by dividing each observed intensity by the estimated sensitivity from the first stage; and

- jointly estimating the parameters in the derived model using the scaled intensity values from the $A L 4, M T 1$, and $C D 1$ data sets.

\subsection{Sensitivity Analysis}

Data sets $S E N S$ and $M T 2$ were used to estimate the sensitivity function, $\psi$, which was approximated by the following second-order polynomial in the momentum coordinates ( $x$ and $y)$ of each pixel:

$$
\psi(x, y ; \mathbf{a}) \approx a_{0}+a_{1} x+a_{2} y+a_{3} x^{2}+a_{4} y^{2}+a_{5} x y
$$

using the observed intensities from $S E N S$. The estimated coefficients in the approximation to $\psi$ were normalized so that $0<\hat{\psi}<1$, where $\hat{\psi}$ is the estimated value of $\psi$ using the least squares estimates of the $a$ 's. Data set $M T 2$ was used to eliminate pixels with unusual observed counts (see Figure 5). Since one would expect these counts to be relatively constant over the range of $x$ and $y$ values, those pixels with $M T 2$ counts $>20$ and also $q^{2}=x^{2}+y^{2}$ values $<0.005$ were omitted from consideration in the estimation of the function $\psi$. In addition, because of the abrupt decline 


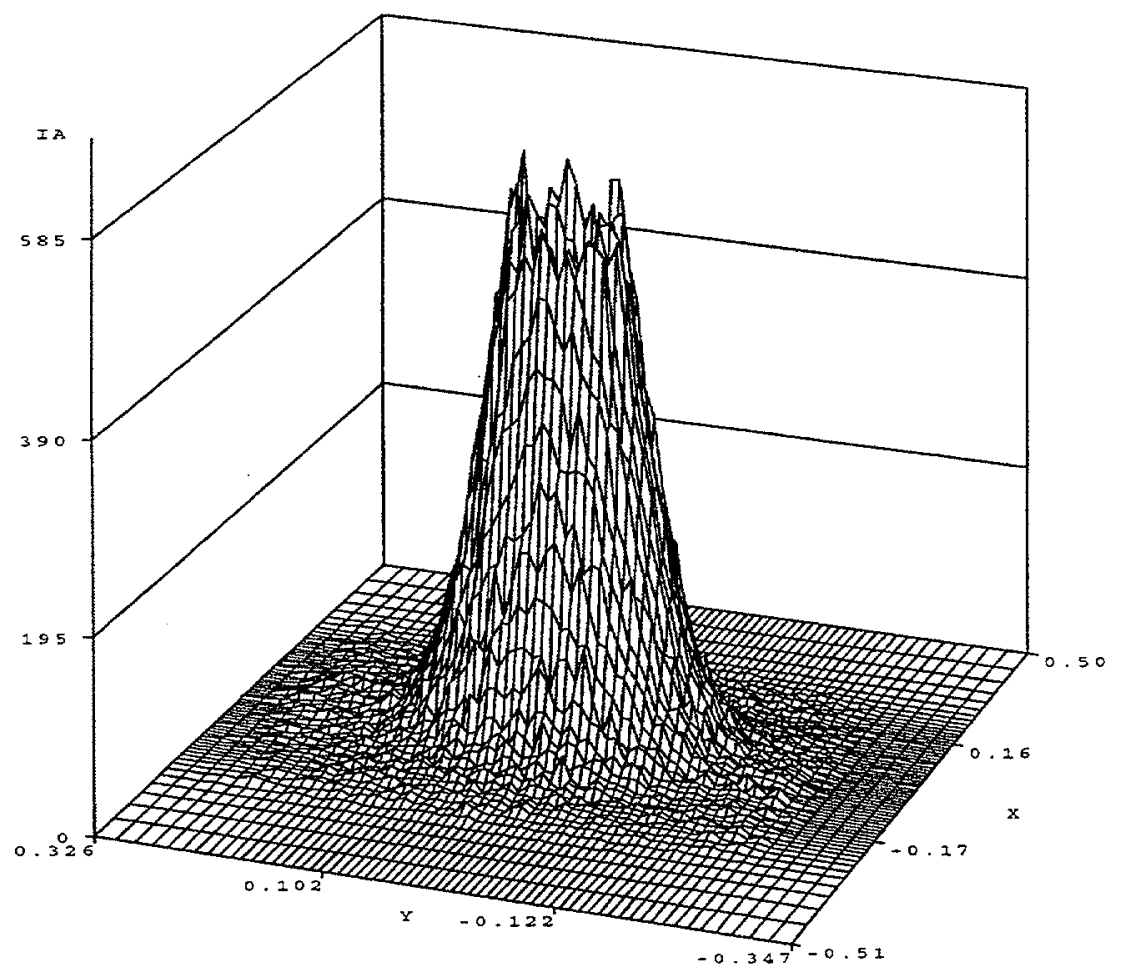

Figure 1: Counts with scatterer.

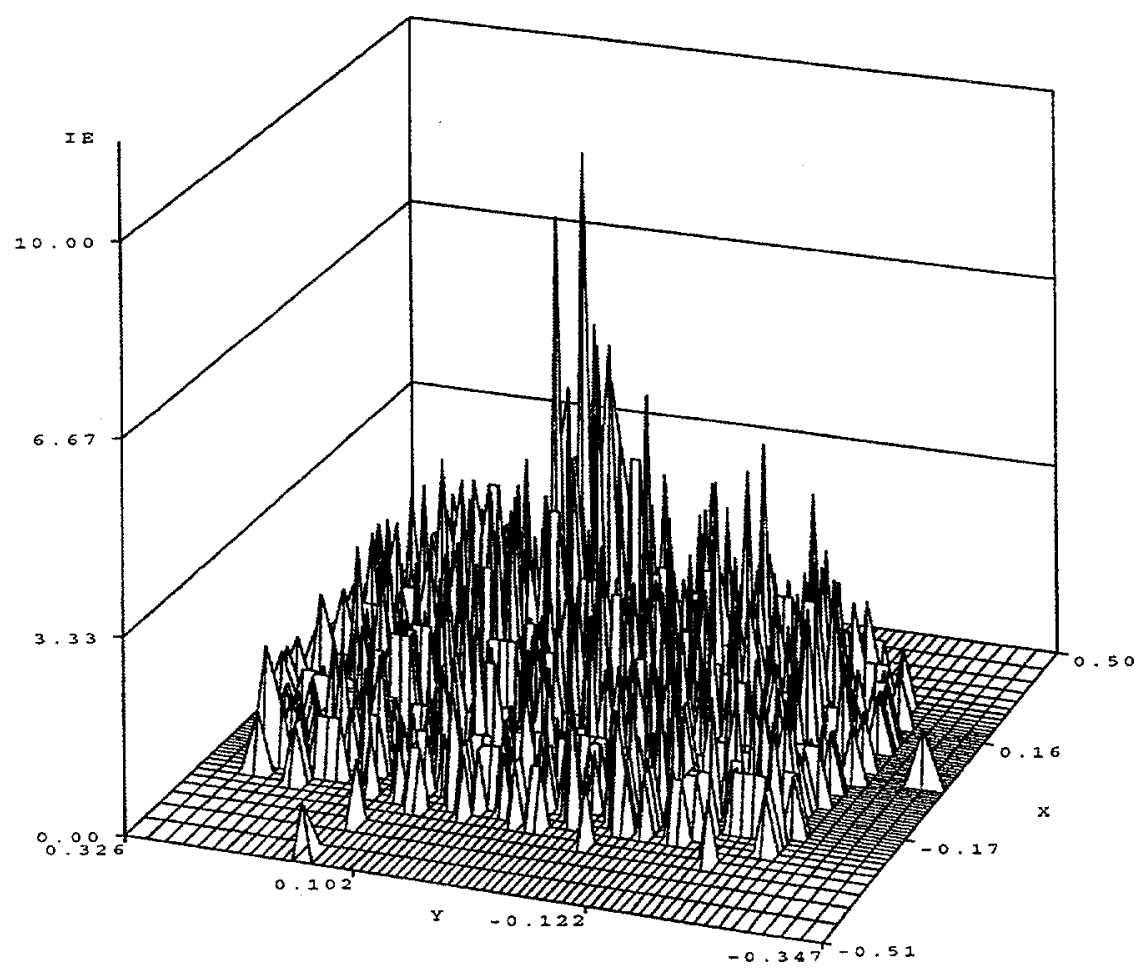

Figure 2: Counts with scatterer removed. 


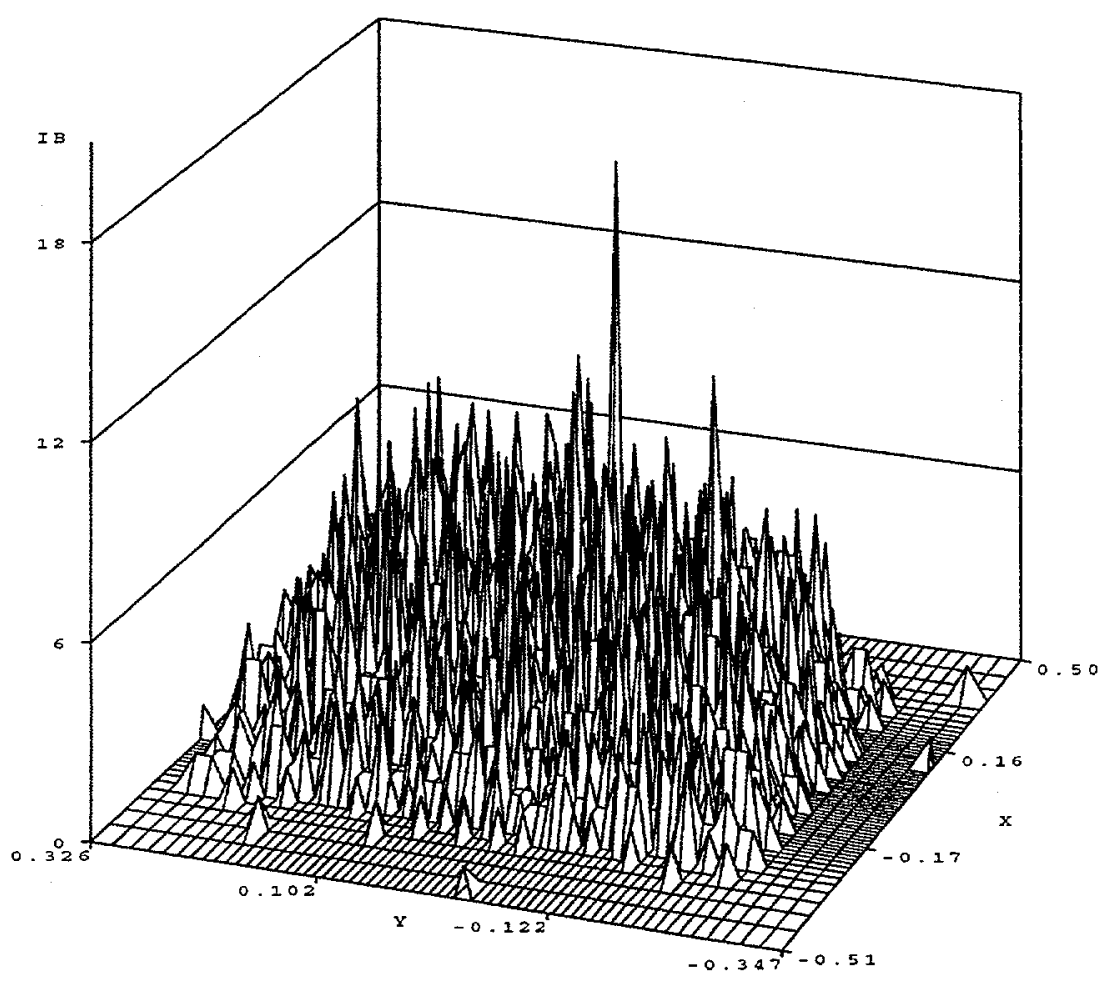

Figure 3: Background counts.

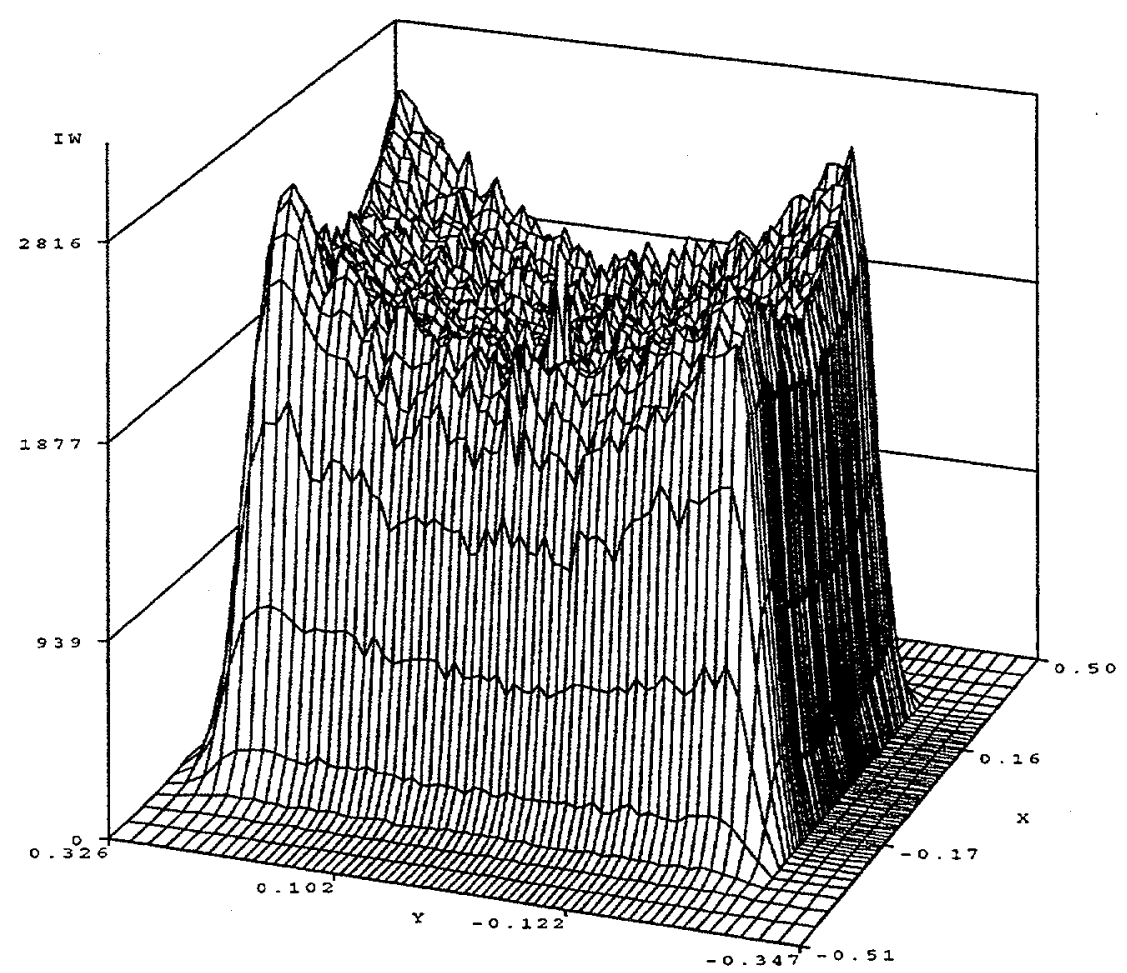

Figure 4: Scattering counts on water target. 


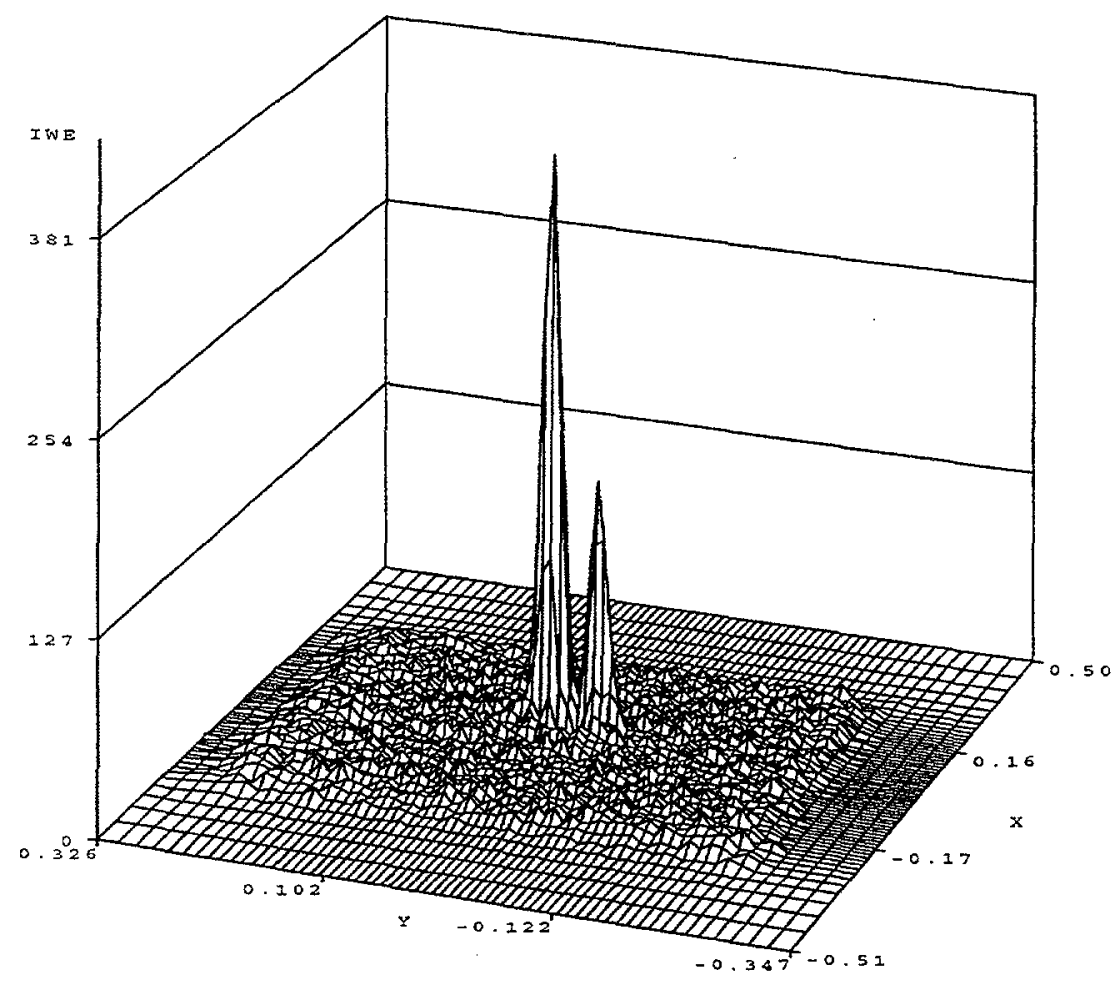

Figure 5: Background counts with the water target removed.

in the sensitivity values at the extreme range of the $x$ and $y$ values, pixels around the edge of the grid were also omitted from consideration. To include the marginal pixels data in the analysis, we would have to replace (11) by more complicated models, e.g., we might use a piecewise polynomial. The range of $x$ and $y$ was defined in order to eliminate the dramatic decline around the edge of the $S E N S$ data (see Figure 4). The selected ranges were $-0.22<x<0.22$ and $-0.22<y<0.20$.

Assuming that the observed counts follow a Poisson distribution, we have the following regression model to analyze

$$
E\left(I_{w} \mid x, y\right)=\operatorname{Var}\left(I_{w} \mid x, y\right)=\psi(x, y ; \mathbf{a}) .
$$

In Appendix 1, the properties of the maximum likelihood estimator and the corresponding numerical algorithms are discussed in detail. Here we only note that the algorithm is based on the reweighted least squares procedure using the NLIN procedure in SAS (1989). The computed estimate, $\hat{\psi}(x, y)=\psi(x, y ; \hat{\mathbf{a}})$, is presented in Figure 6 . The blank grid elements correspond to outliers detected in the background noise measurements. Because of the orientation of this plot, it is not possible to see additional blanks in the vicinity of the center. Note that the standard error of $\hat{\psi}(x, y)$ changes from 0.00086 to 0.00245 . These numbers are significantly less than the standard errors ( 0.0153 and 0.0198 , respectively) for the raw $I_{w}$ that are usually recommended for data corrections [see Chen and Lin (1987)].

\subsection{Fitting the Compound Model}

From the physics of observed counts $I_{a}, I_{e}$, and $I_{b}$ (see also Figures 1-3), it is clear that each must be described with its own model. To combine all three into one response surface model [see Box 


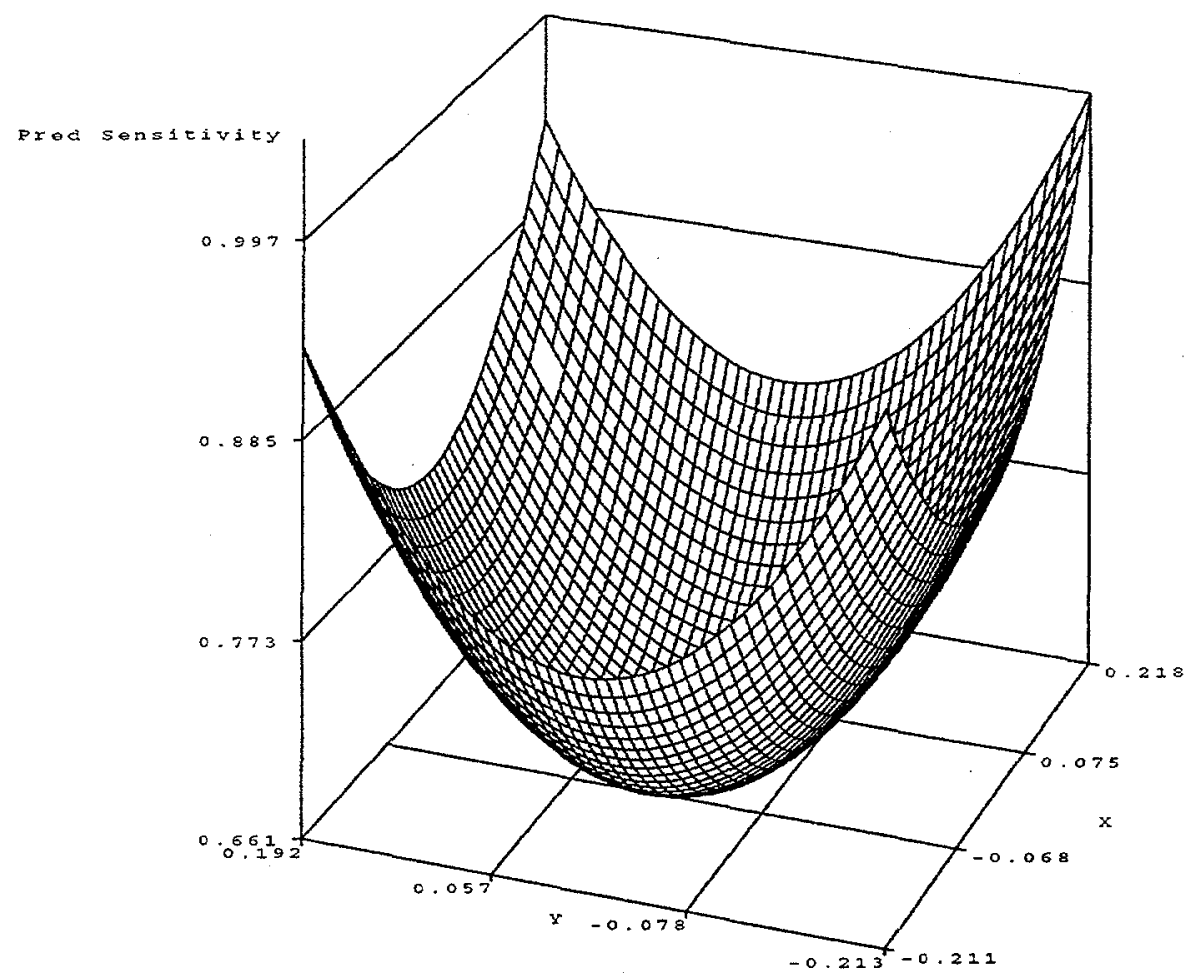

Figure 6: Estimated sensitivity function $\hat{\psi}(x, y)$.

and Draper (1987)], we propose the following

$$
\begin{array}{r}
\eta(q, z, t ; \theta)=\eta_{1}\left(q, \theta_{1}\right) t_{1} z_{1} z_{2}+\eta_{2}\left(q, \theta_{2}\right)\left[t_{1} z_{1} z_{2}+t_{2}\left(1-z_{1}\right) z_{2}\right] \\
+\eta_{3}\left(q, \theta_{3}\right)\left[t_{1} z_{1} z_{2}+t_{2}\left(1-z_{1}\right) z_{2}+t_{3}\left(1-z_{1}\right)\left(1-z_{2}\right)\right]
\end{array}
$$

where $\theta^{T}=\left(\theta_{1}{ }^{T}, \theta_{2}{ }^{T}, \theta_{3}{ }^{T}\right), \eta_{1}\left(q, \theta_{1}\right)$ models the scattering by the aluminum target, $\eta_{2}\left(q, \theta_{2}\right)$ is the model for the empty cell scattering, and $\eta_{3}\left(q, \theta_{3}\right)$ is the noise or background signal model. The symmetry with respect to rotation is assumed everywhere and, therefore, all models depend only upon the momentum value $q=\sqrt{x^{2}+y^{2}}$. The multipliers $t_{1}, t_{2}$, and $t_{3}$ are the times used to measure $I_{a}, I_{e}$, and $I_{b}$, respectively. The $z$ 's are dummy variables such that $\left(z_{1}, z_{2}\right)=(1,1)$, $\left(z_{1}, z_{2}\right)=(0,1)$, and $\left(z_{1}, z_{2}\right)=(0,0)$ when $I_{a}, I_{e}$, and $I_{b}$ are correspondingly measured. We assume that the observed counts have a Poisson distribution, and

$$
E(I \mid q, z, t ; \theta)=\eta(q, z, t ; \theta) .
$$

If the neutron source has an intensity that varies in time, then the times $t_{1}, t_{2}$, and $t_{3}$ must be replaced by the monitor counts. The replacement is valid in the framework of (12) and (13) only if the monitor counts are large. Otherwise, we must consider their randomness and introduce models describing this randomness. For instance, if, at all pixels, the registered counts have a Poisson distribution, then normalization by the monitor counts leads to the necessity of considering the conditional distribution of counts given the overall counts (which are proportional to the monitor counts). This conditional distribution is multinomial [see Johnson and Kotz (1969), Chapt. 4.4]

Model (14) describes the number of scattered neutrons given $q, z$, and $t$. To model the number of registered particles, we can either inflate the observed data dividing them by the efficiency function 
$\psi(q)$ and by the transparency coefficient $\gamma$, or multiply correspondingly the function $\eta(q, z, t ; \theta)$. Theoretically, both procedures are equivalent. We used the first adjustment. The mathematical background to use "inflation" may be found in Johnson and Kotz [(1969), Chapter 8.2], where the compound Poisson-binomial distribution is discussed in detail.

Following the technique presented in Appendix 1, we use the reweighted least squares method of estimation with the SAS NLIN procedure [SAS (1989)] to obtain the maximum likelihood estimates of the $\theta$ 's. The weighting function $w(q, z, t ; \theta)$ for this model is given by $\psi(q)\left[\gamma z_{1} z_{2}+1-z_{1}\right] / \eta(q, z, t ; \theta)$, since the actual observations for the $I_{a}$ and $I_{e}$ data sets can be related to a compound Poissonbinomial distribution as mentioned earlier.

We have considered three alternatives for model (13). In all of these alternatives, $\eta_{2}\left(q, \theta_{2}\right)$ and $\eta_{3}\left(q, \theta_{3}\right)$ were approximated by first degree polynomials in $q^{2}$. The first component of $\eta_{1}\left(q, \theta_{1}\right)$ was subsequently [compare with (7) and (8)] selected as

- the Guinier model

$$
\theta_{11} e^{-\theta_{12} q^{2}}
$$

- the Rayleigh model

$$
\theta_{11}\left[\frac{\sin \left(\theta_{12} q\right)-\theta_{12} q \cos \left(\theta_{12} q\right)}{\left(\theta_{12} q\right)^{3}}\right]^{2}
$$

and

- the two-exponential model

$$
\theta_{11} e^{-\theta_{12} q^{2}}+\theta_{13} e^{-\theta_{14} q^{2}}
$$

We have used all observations satisfying the following: $\gamma=0.95 ; q^{2}>0.005 ;-0.22<x<0.22 ;$ and $-0.22<y<0.20$. For the first and third alternatives, $R_{g}=\sqrt{3 \theta_{12}}$, and for the second alternative $R_{g}=\theta_{12} \sqrt{3 / 5}$. Figures $7-9$ are scatter plots of the residuals [i.e., (observed - predicted) versus momentum] for all three alternative models. Only the plot in Figure 9 shows no systematic bias patterns within the cone-shape scatter of residuals that is typical for Poisson distributed data. The presence of patterns, which are seen in Figures 7 and 8, indicates some bias in the model being used to describe the dependence.

The values of

$$
v^{2}=\sum_{i=1}^{n} w\left(q_{i}, z_{i}, t_{i} ; \hat{\theta}\right)\left(I_{i}-\eta\left(q_{i}, z_{i}, t_{i} ; \hat{\theta}\right)\right)^{2}
$$

for the above models are $1.306,2.039$, and 1.115 , respectively. Theoretically, $v^{2}$ must be close to 1 for the "good" models. These numbers indicate that (17) is the best choice. To our knowledge, the negligence of the following facts can lead to the noticeable systematic deviation of the physically sound models (15) and (16) from the observed signal:

- nonzero probability of secondary scattering;

- smoothing effect of finite sized pixels and the neutron collimation system;

- random nature of the radius of gyration, $R_{g}$; and

- relatively wide range for $q$ [this is especially important for (15) which is valid only asymptotically when $\theta_{12} q^{2} \rightarrow 0$ (see Section 1$\left.)\right]$. 


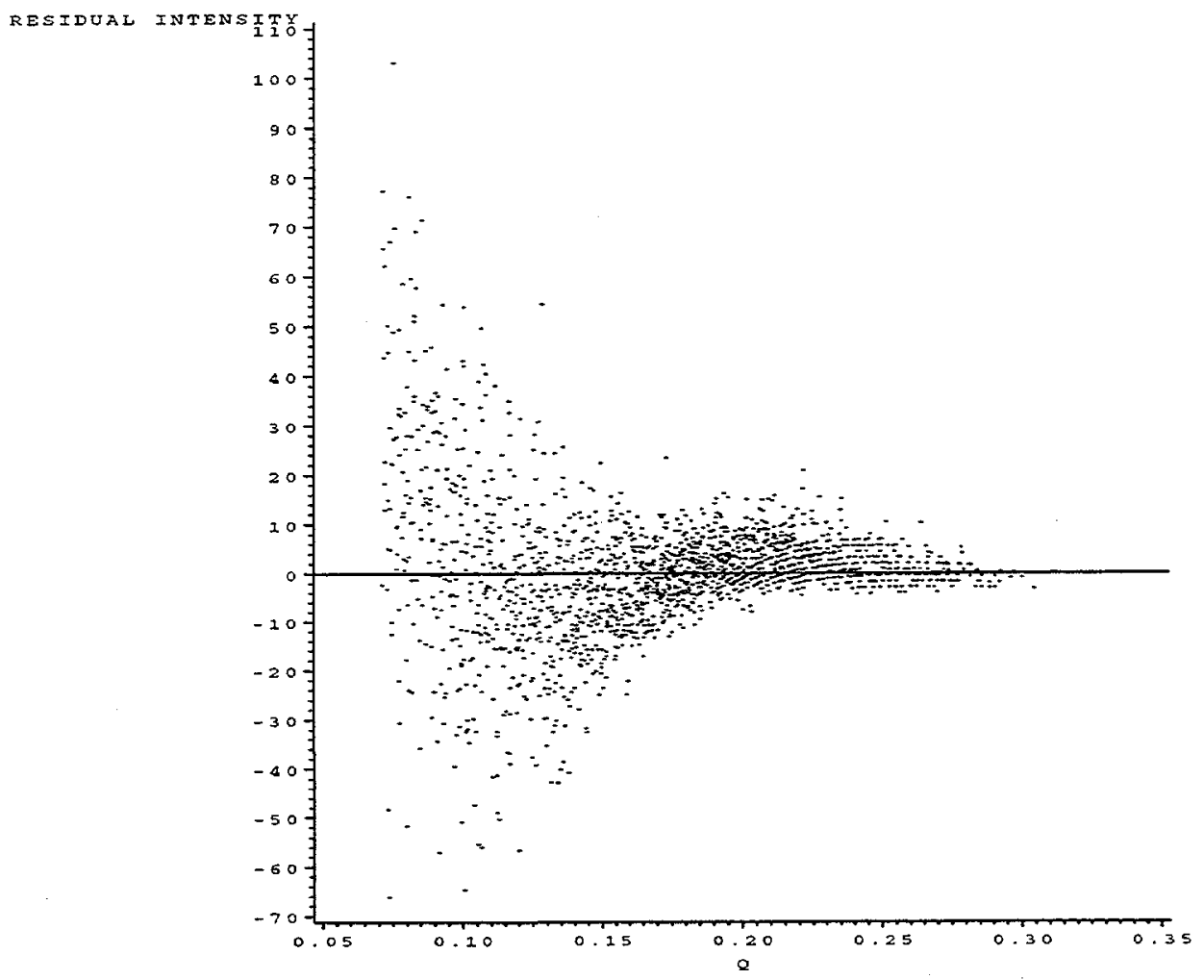

Figure 7: Residual vs momentum for the Guinier model.

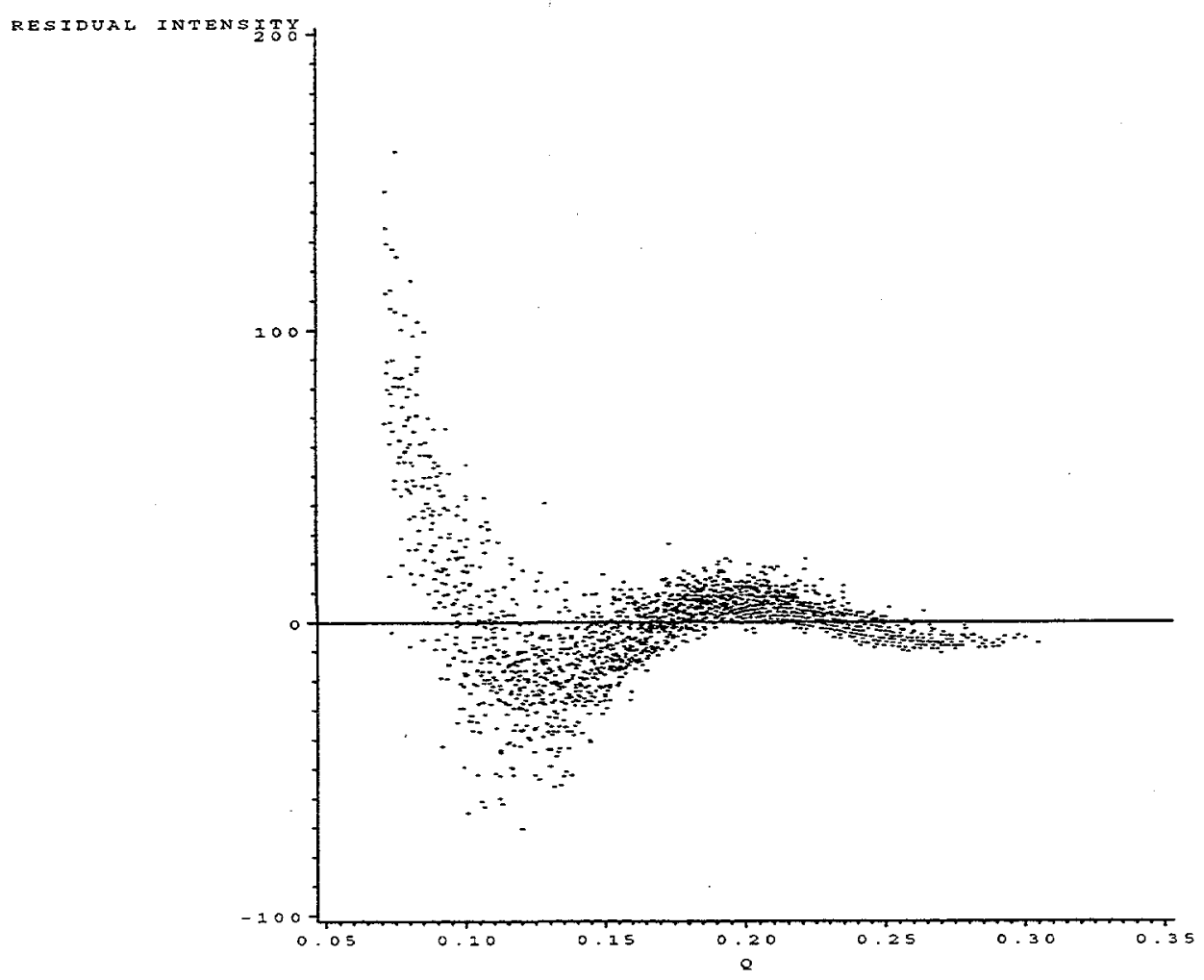

Figure 8: Residual vs momentum for the Rayleigh model. 


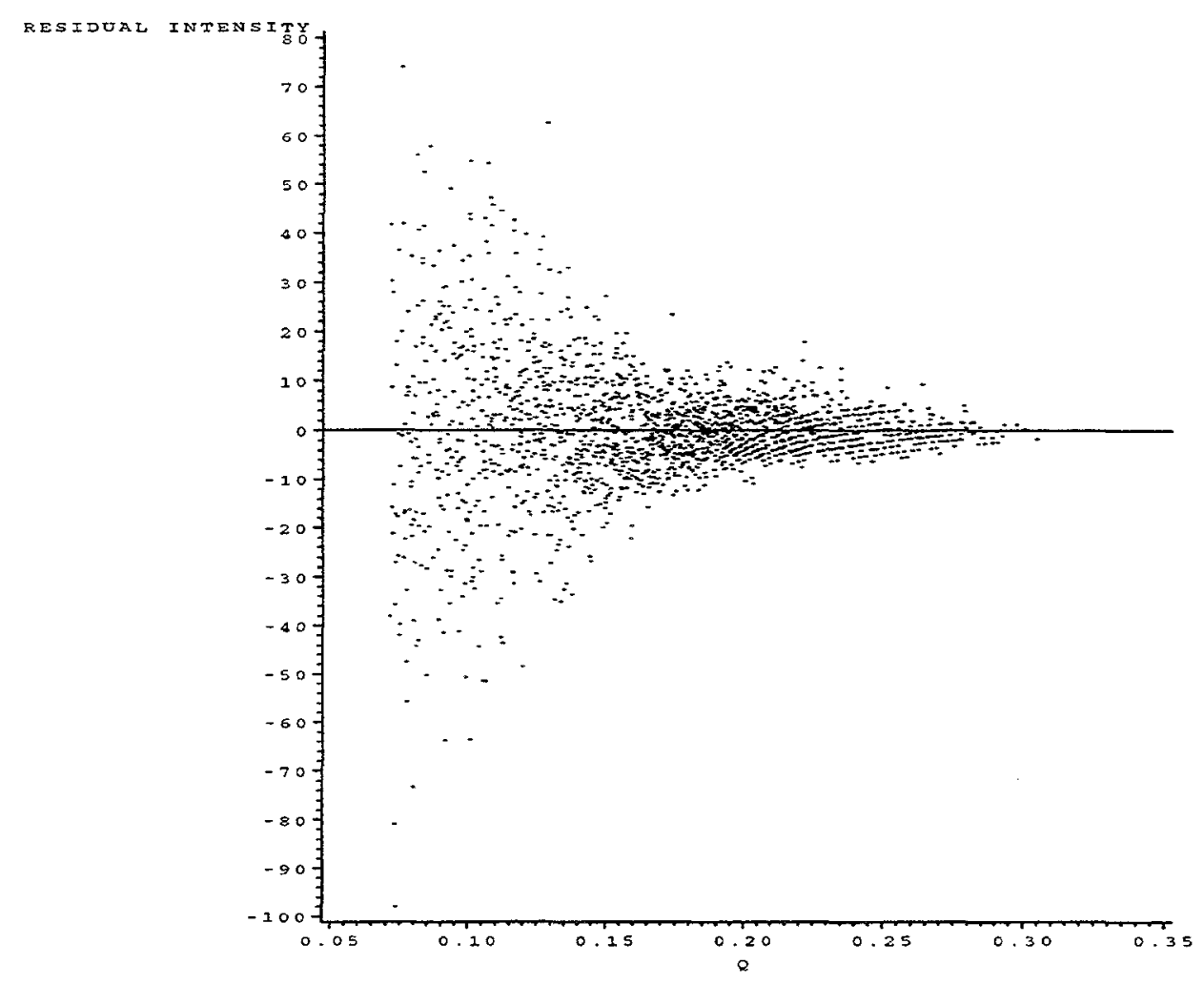

Figure 9: Residual vs momentum for the two-exponential model.

The following should be noted:

- Using the Taylor expansion we can find the correction terms that must be added to observations in an iterative manner. The randomness of $R_{g}$ results in the correction term

$$
\Delta I_{a}=\frac{1}{2} \operatorname{Var}\left(R_{g}\right) q^{2} \eta_{1}^{\prime \prime}\left(\tau, \theta_{1}\right)+O\left(q^{3} \mu_{3}\right)
$$

where $\tau=R_{g} q, \eta_{1}^{\prime \prime}$ stands for the second derivative of $\eta_{1}$ with respect to $\tau$ and $\mu_{3}$ is the third central moment of the probability density of $R_{g}$.

- The smearing effect caused by the finite number of pixels leads to the following [compare, for instance, with Wignall et al. (1988)]:

$$
\Delta I_{a}=\frac{1}{2} R_{g}^{2}(\widehat{\Delta q})^{2} \eta_{1}^{\prime \prime}\left(\tau, \theta_{1}\right)+O\left(R_{g}^{4}\left(\widehat{\Delta q)^{4}}\right)\right.
$$

where $\left(\widehat{\Delta q}^{k}\right.$ is the average value of $(\Delta q)^{k}$ over an area associated with a given pixel; that area is assumed symmetrical with respect to $\tau$. In iterative calculations similar to those proposed in A.1, the true parameter may be replaced by estimates from the previous iteration. Note, that the similar correction formulae may be derived to describe the neutron collimation system smearing effects.

In the remainder of this section we present the detailed statistical information only for model (17). Table 1 gives a summary of the parameter estimates for this model with $\gamma=0.95$, and the previously mentioned restrictions of $x, y$, and $q^{2}$. Figure 10 shows the exponential decline in the 
Table 1: Parameter estimates

$\left(\gamma=0.95 ; q^{2}>0.005 ;-0.22<x<0.22 ;-0.22<\right.$
\begin{tabular}{|c||cc|}
\hline Parameter & Estimate & Std. Err. \\
\hline$\theta_{11}$ & 62.618 & 0.5244 \\
$\theta_{12}$ & 142.885 & 1.0837 \\
$\theta_{13}$ & 0.7885 & 0.1013 \\
$\theta_{14}$ & 20.285 & 2.4119 \\
$\theta_{20}$ & 0.01905 & 0.006255 \\
$\theta_{21}$ & -0.2582 & 0.1392 \\
$\theta_{30}$ & 0.1395 & 0.002582 \\
$\theta_{31}$ & -1.2880 & 0.05862 \\
\hline
\end{tabular}

AL4 intensities as a function of $q^{2}$ along with the fitted values of $\eta_{1}$. This provides an overall visual evaluation of the model adequacy. Figure 11 is a plot of the observed versus the predicted $A L 4$ intensities along with the 1:1 line for reference. The observed pattern about this line reveals a well-behaved fitted model. Figures 7-11 are frequently used visual aids to evaluate the proposed model adequacy in describing the observed data, and, generally, these plots reveal a fitted model that does quite well in describing the variation in the observed intensities. Additional residual plots for the $M T 1$ and $C D 1$ data sets are given in Figures 12-13. The layered pattern in these residual plots is expected because of the integer nature of the data.

Statistically, the considered model fits data adequately. However, the estimated value of $\hat{R}_{g}=$ $\sqrt{3 \hat{\theta_{12}}}=20.7 \mathrm{~nm}$ is significantly (in the statistical sense) greater than the commonly accepted value $R_{g}=20.2 \mathrm{~nm}$ [Wignal and Bates (1987)]. A possible explanation of that may be the phenomena described by (19) and (20). We fitted model (17) with $\theta_{12}$ and $\theta_{14}$ fixed at $136.0 \mathrm{~nm}$ and $68.0=\theta_{12} / 2$ correspondingly. The value of $\theta_{12}$ was selected to match $R_{g}=20.2 \mathrm{~nm}$. The chosen relationship between $\theta_{12}$ and $\theta_{14}$ is based on the assumption that there exists a noticeable secondary neutron scattering.

Considering two consecutive scatterings as adding two independent and identically distributed random variables we may conclude that $2 \theta_{14}=\theta_{12}$ [the variance of the sum of independent random variables equals the sum of their variances; for the more detailed analysis see Schelten and Schmatz (1980)]. Figure 14 shows that the fitted function is going slightly above the data point cloud wherever $\eta^{\prime \prime}(\tau)<0$; compare with Figure 10. This fact agrees with (19) and (20). Note that $\theta_{11}=53.8$ and $\theta_{14}=3.4$, i.e., about $6 \%$ of the total number of scattered neutrons is attributed to double scattering. This number is higher than $\sim 1 \%$ that we derived from Schelten and Schmatz (1980).

In the appendix we explain why the use of the compound model (13) is more preferable than the stage-wise analysis of the scattering data with the target, cadmium shield, and the empty cell. In short, the use of a compound model may be considered as a more sophisticated handling of the available information. However, that sophistication results in more complicated computations, and we have to be cautious when the total number of unknown parameters becomes large. It is much easier to minimize in two-dimensional space (model $\eta_{3}$, describing background noise), then again in two-dimensional space (model $\eta_{2}$, describing scattering on empty cell), and, finally, in threedimensional space (model $\eta_{1}$ ), describing scattering on the aluminum target). The pixel-wise data correction, which is common in SANS [see, for example, Chen and Lin (1987), Section 16.2.2.3], is 


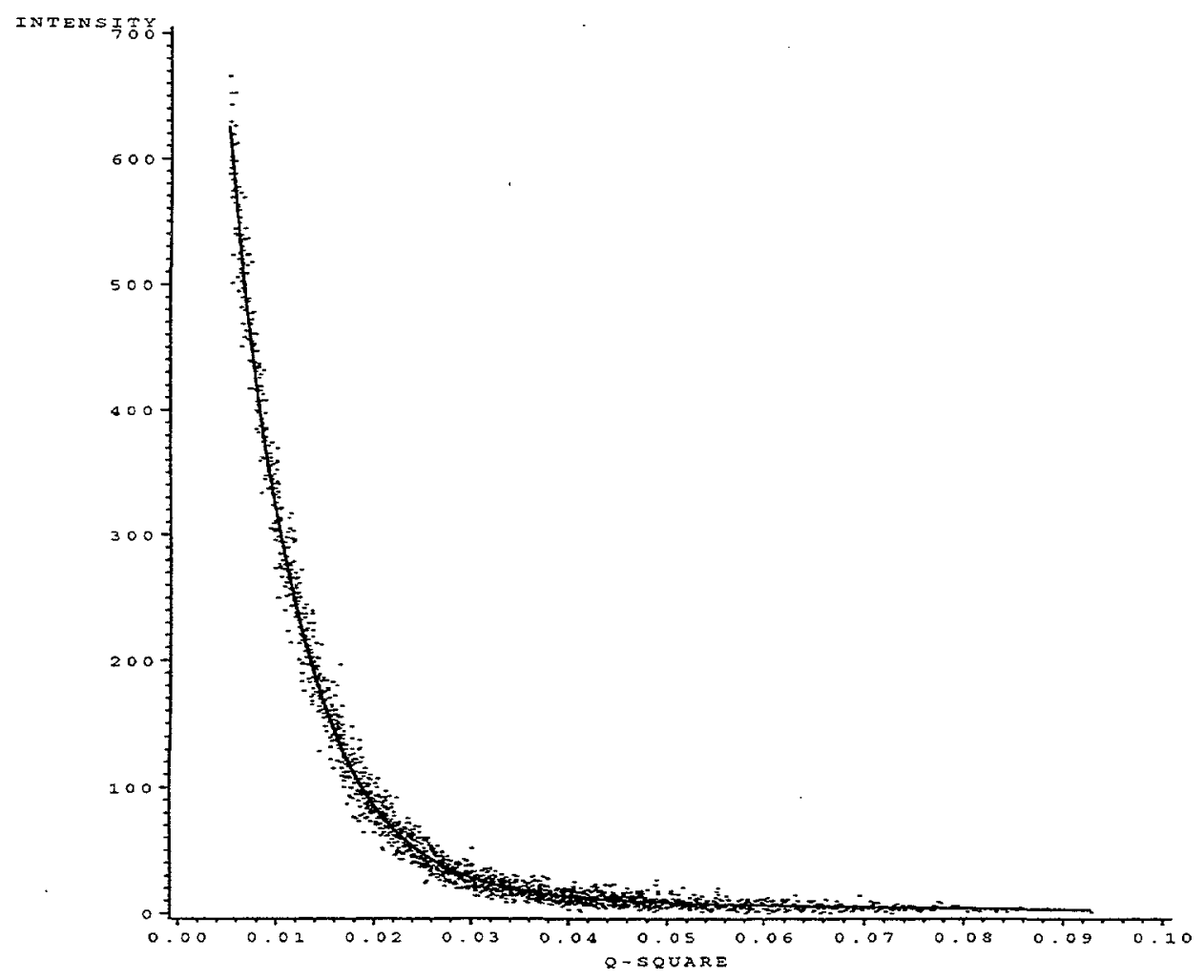

Figure 10: Data and fitted model $(17)$ for $\eta_{1}\left(q^{2}\right)$.

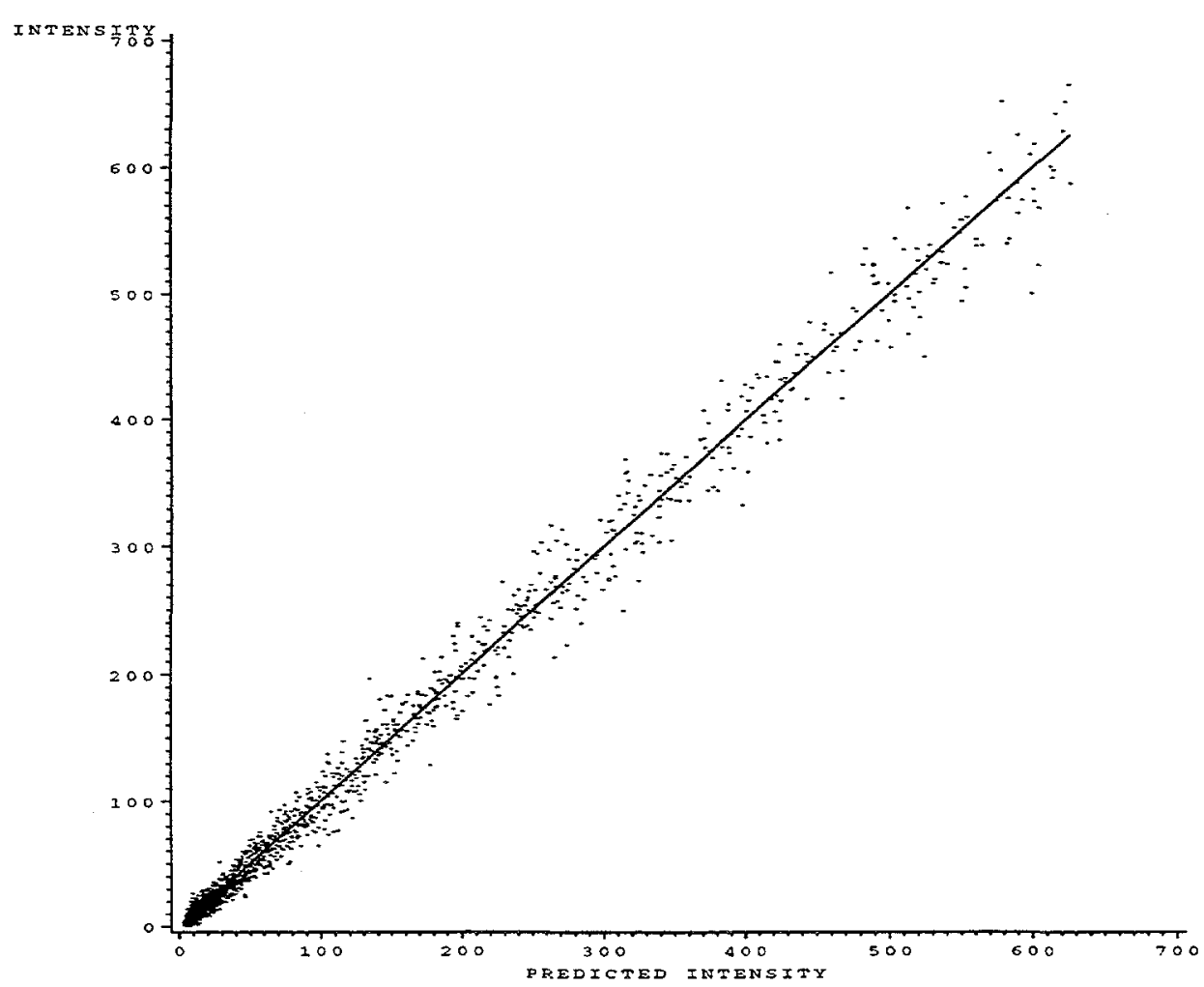

Figure 11: Obsered vs predicted intensity for model (17) as $\eta_{1}\left(q^{2}\right)$. 


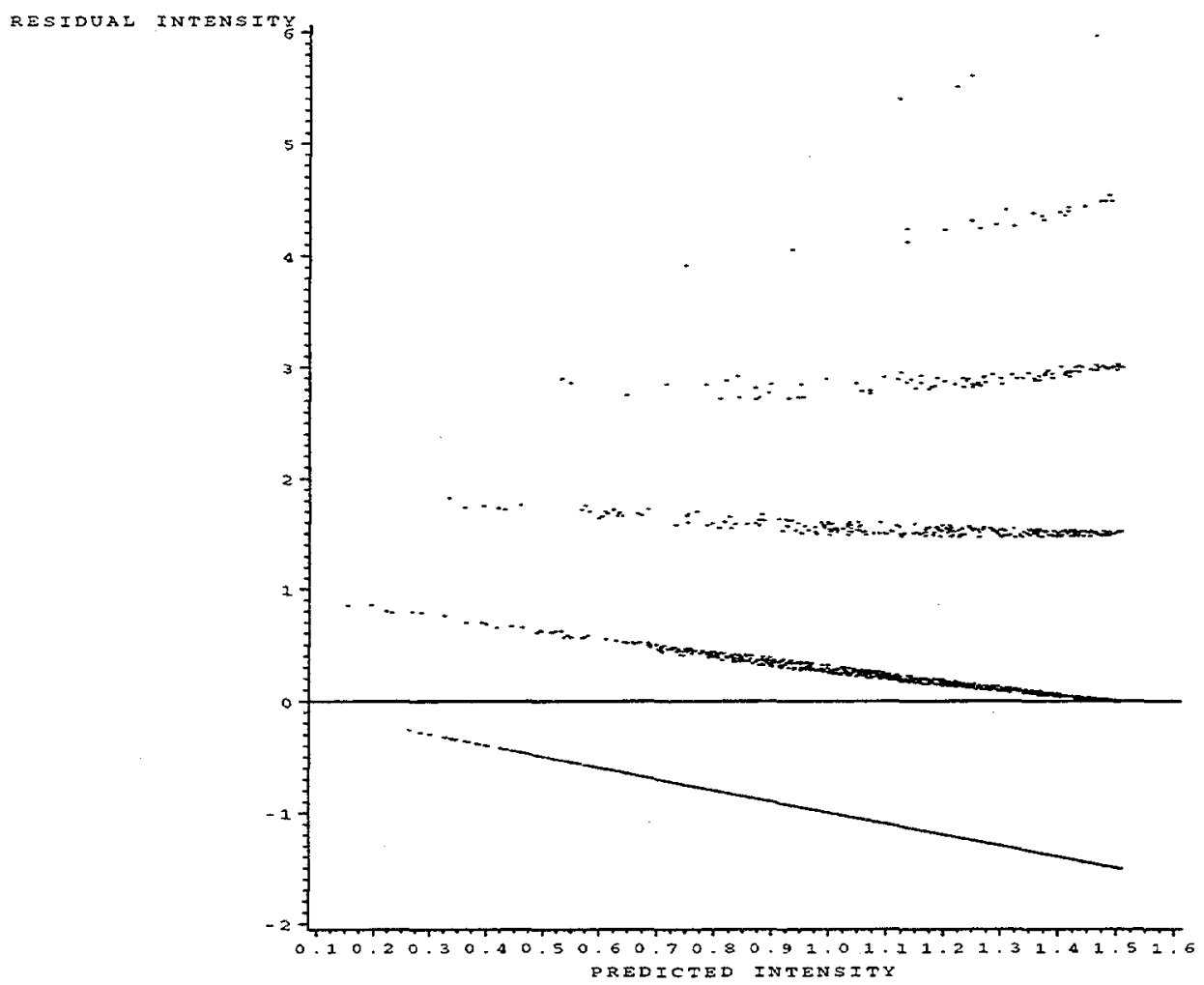

Figure 12: Residual vs predicted intensity for $\eta_{2}\left(q^{2}\right)$ combined with (17).

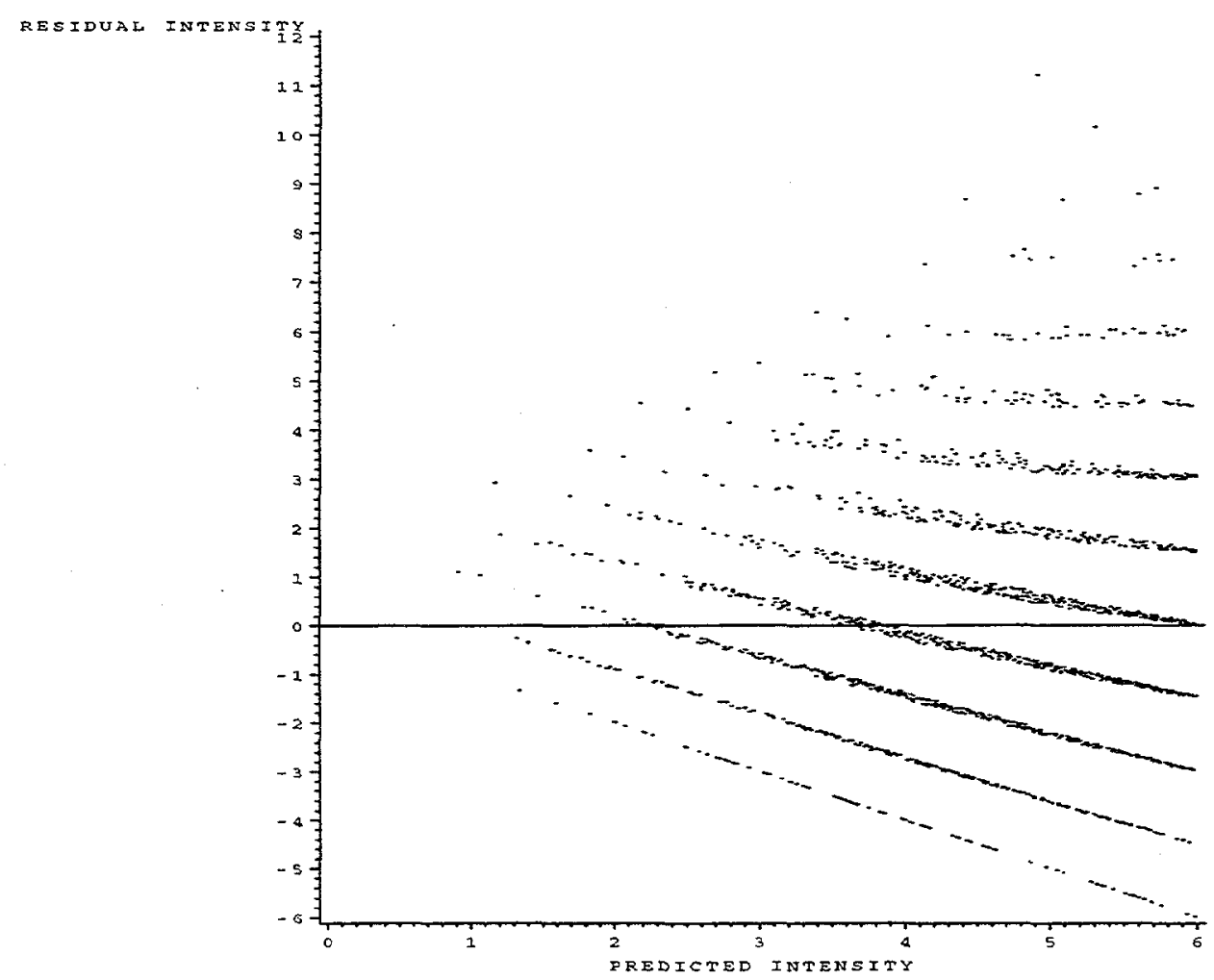

Figure 13: Residual vs predicted intensity for $\eta_{3}\left(q^{2}\right)$ combined with (17). 


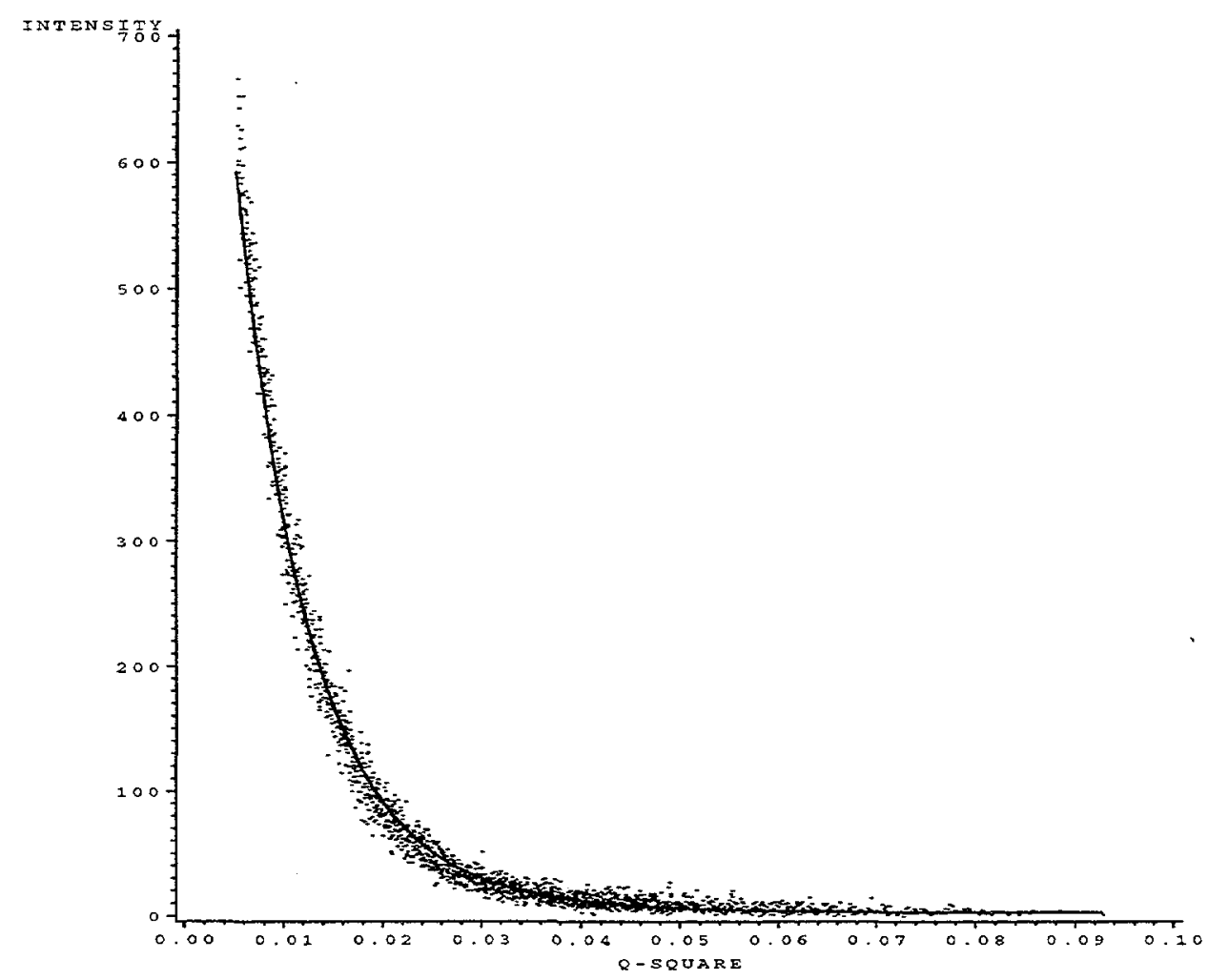

Figure 14: Data and fitted model (17) for $\eta_{1}\left(q^{2}\right)$ with $\theta_{12}=2 \theta_{14}=136.0 \mathrm{~nm}$.

a particular case of consecutive analysis with a number of unknown parameters at each of the first two stages (background noise and scattering on empty cell) being equal to the number of pixels. There is one positive thing about the latter approach, namely, we do not need any models (see our $\eta_{2}\left(q, \theta_{2}\right)$ and $\left.\eta_{3}\left(q, \theta_{3}\right)\right)$ to describe the neutron counts. Hence, no systematic bias, due to the wrong selection of model(s), would appear in the estimators. However, the presence of a large number of nuisance parameters can lead to a drastic increase in the variance of the estimators of the main set of parameters.

\section{ROBUSTNESS OF ESTIMATORS}

From now on, our discussion is based mainly on model (17). As an additional consideration of the effect of the choice of $\gamma$ and the $q^{2}$ restriction on the parameter estimates, the parameters were also estimated for the following choices of these conditions:

$$
\begin{aligned}
& \gamma=1.00, \quad q^{2}>0.003 \\
& \gamma=1.00, \quad q^{2}>0.007 \\
& \gamma=0.90, \quad q^{2}>0.003 \\
& \gamma=0.90, \quad q^{2}>0.007
\end{aligned}
$$

Table 2 is a summarization of the parameter estimates for these four combinations plus the combination of $\gamma=0.95$ and $q^{2}>0.005$; in all cases $-0.22<x<0.22$ and $-0.22<y<0.20$. We shall focus on the parameter of prime interest, $\theta_{12}$. Although the parameter estimates did not 
Table 2: Parameter estimates from robustness study

\begin{tabular}{|c|cc|c|cc|}
\hline Parameter & \multicolumn{2}{|c|}{$q^{2}>0.003$} & $q^{2}>0.005$ & \multicolumn{2}{c|}{$q^{2}>0.007$} \\
& $\gamma=0.90$ & $\gamma=1.00$ & $\gamma=0.95$ & $\gamma=0.90$ & $\gamma=1.00$ \\
\hline$\hat{\theta}_{11}$ & 65.835 & 59.251 & 62.618 & 67.339 & 60.591 \\
Std. Err. & 0.3757 & 0.3380 & 0.5244 & 0.8258 & 0.7425 \\
$\hat{\theta}_{12}$ & 142.468 & 142.420 & 142.885 & 144.600 & 144.536 \\
Std. Err. & 0.8708 & 0.8691 & 1.0837 & 1.3800 & 1.3800 \\
$\hat{\theta}_{13}$ & 0.8101 & 0.7031 & 0.7885 & 0.9412 & 0.8220 \\
Std. Err. & 0.09628 & 0.08654 & 0.1013 & 0.1246 & 0.1128 \\
$\hat{\theta}_{14}$ & 19.603 & 19.476 & 20.285 & 22.044 & 22.021 \\
Std. Err. & 2.2527 & 2.3409 & 2.4119 & 2.4668 & 2.5676 \\
$\hat{\theta}_{20}$ & 0.02331 & 0.02336 & 0.01905 & 0.01623 & 0.01629 \\
Std. Err. & 0.006004 & 0.006001 & 0.006255 & 0.006534 & 0.006530 \\
$\hat{\theta}_{21}$ & -0.3276 & -0.3289 & -0.2582 & -0.2101 & -0.2116 \\
Std. Err. & 0.1339 & 0.1338 & 0.1393 & 0.1447 & 0.1445 \\
$\hat{\theta}_{30}$ & 0.1402 & 0.1402 & 0.1395 & 0.1390 & 0.1390 \\
Std. Err. & 0.002464 & 0.002464 & 0.002582 & 0.002706 & 0.002706 \\
$\hat{\theta}_{31}$ & -1.3010 & -1.3010 & -1.2880 & -1.2776 & -1.2776 \\
Std. Err. & 0.05653 & 0.05653 & 0.05862 & 0.06078 & 0.06078 \\
\hline
\end{tabular}

change drastically across these combinations, there was an indication that the standard errors on the parameter estimates increased as $q^{2}$ went from 0.003 to 0.007 .

The increase in the standard errors is a simple reflection of the statistical fact that the standard error of any reasonable estimator is decreasing, if the number of observations included in the analysis increases and the underlying model is valid (i.e., the bias is much less than the standard errors of the observations). One may recollect, for instance, that the standard error of the arithmetic

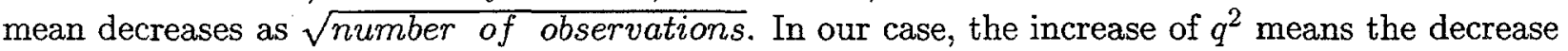
in the number of observations, which results in the increase of the standard error. Thus, the recommendation is to enlarge the number of pixels (i.e., to decrease $q^{2}$ ) until the model is still valid and there is no indication of the presence of counts generated by the direct neutron beam. The estimate of the most interesting parameter, $\theta_{12}$, varies noticeably, and it is getting worse for large $q^{2}$ when we leave the area in which the Guinier model works best. Note that the reference value for $\theta_{12}=R_{g}^{2} / 3 \simeq 136.0 \mathrm{~nm}$ according to [Wignall and Bates (1987)]. Table 3 gives the parameter estimates for model (15) and the same restrictions as in Table 1. The most signigficant change in the parameter estimates for this reduced model when compared with the similar parameters in the full model (Table 1), is the change in sign and magnitude of the $\theta_{20}$ and $\theta_{21}$ parameters. This result again follows from the poor ability of the Guinier model to approximate the data for large $q^{2}$ and the fact that the function $\eta_{2}\left(q^{2}\right)$ "tries" to compensate for this. 
Table 3: Parameter estimates for reduced model

$$
\left(\gamma=0.95 ; q^{2}>0.005 ;-0.22<x<0.22 ;-0.22<y<0.20\right)
$$

\begin{tabular}{|c||cc|}
\hline Parameter & Estimate & Std. Err. \\
\hline$\theta_{11}$ & 55.658 & 0.3444 \\
$\theta_{12}$ & 126.560 & 0.4424 \\
$\theta_{20}$ & -0.01333 & 0.006659 \\
$\theta_{21}$ & 2.1785 & 0.1538 \\
$\theta_{30}$ & 0.1395 & 0.002582 \\
$\theta_{31}$ & -1.2880 & 0.05862 \\
\hline
\end{tabular}

A comparison of Table 1 and Table 3 reveals a significant drop in value of $\hat{\theta}_{12}$. It can be explained by any of the causes discussed in the previous section, but most probably by the fact that model (15) is valid only for the small momentum, $q$. It is also expedient to analyze how variations of the upper extreme of $q^{2}$ may affect the estimate of the parameter $\theta_{12}$. The range on $q^{2}$ was defined by $0.005<q^{2}<q_{\max }^{2}$ with $q_{\max }^{2}=0.01$ to 0.04 in steps of 0.005 . Table 4 provides a summary of the $\theta_{12}$ estimates for models (15) and (17) as a function of $q_{\max }^{2}$ when $\gamma=0.95$. The value of $q^{2}=0.097$ corresponds to the restrictions from Table 1.

From a review of Table 4, the following conclusions/observations may be made:

- The single exponential model for $\eta_{1}$ did not have numerical estimation problems across the whole range of $q_{\max }^{2}$ values.

- The double exponential model had numerical estimation problems (singular Hessian matrix) for $q_{\max }^{2}<0.020$. However, the point estimates of $\theta_{12}$ at these values of $q_{\max }^{2}$ were equal to the $\theta_{12}$ estimates for the single exponential model. This means that the $\theta_{13}$ component was automatically zeroed out.

- Since $v^{2}$ in (18) is asymptotically $(n \rightarrow \infty)$ normally distributed with standard deviation $\sigma_{v}=\sqrt{\operatorname{Var}\left(v^{2}\right)} \simeq \sqrt{2 /(n-m)}$, where $m$ is the number of estimated parameters, one can find out that for the Guinier model (the upper part of Table 4) the $v^{2}$ values are beyond the $3 \sigma_{v}$ level for all $q_{\max }^{2} \geq 0.025$. For the two-exponential model, only the case with $q_{\max }^{2}=0.025$ has suspiciously large values of $v^{2}$.

- The estimates for the standard errror of $\hat{\theta}_{12}$ for the Guinier model must be considered very cautiously starting with $q_{\text {max }}^{2}=0.020$. This is because of the poor approximation of "tail" observations by that model with extremely low predicted values of $\hat{\eta}(q)$ that lead to exaggerated weights and consequently artificially low estimates of the standard error of $\hat{\theta}_{12}$. See also equation (29) in the appendix.

- As $q_{\text {max }}^{2}$ increases, the estimates of $\theta_{12}$ diverge from the expected value of 136 . For the double exponential model, the large values of $q_{\max }^{2}$ appear to produce over estimates of $\theta_{12}$.

As an additional consideration, we compared the estimates of $\theta_{12}$ in the following models for $\eta_{1}\left(q, \theta_{1}\right)$ :

$$
\begin{gathered}
\theta_{11} \phi\left(\theta_{12} q\right), \\
\theta_{11} \phi\left(\theta_{12} q\right)+\theta_{13} \phi\left(\theta_{14} q\right), \\
\theta_{11} \phi\left(\theta_{12} q\right)+\theta_{13} \phi\left(\theta_{12} q / \sqrt{2}\right),
\end{gathered}
$$


Table 4: Estimates of $\theta_{12}$ for $\gamma=0.95,0.005<q^{2}<q^{2}$ max

\begin{tabular}{|c|c|c|c|c|}
\hline$\eta_{1}\left(q, \theta_{1}\right)=\theta_{11} e^{-\theta_{12} q^{2}}$ \\
\hline 0.010 & 579 & 135.71 & 3.0461 & 0.988 \\
0.015 & 1152 & 135.73 & 1.2808 & 1.056 \\
0.020 & 1710 & 135.21 & 0.8318 & 1.078 \\
0.025 & 2259 & 134.04 & 0.6484 & 1.094 \\
0.030 & 2808 & 132.68 & 0.5543 & 1.089 \\
0.035 & 3354 & 130.80 & 0.5009 & 1.094 \\
0.040 & 3846 & 129.01 & 0.4732 & 1.124 \\
0.097 (see text) & 5589 & 126.56 & 0.4424 & 1.306 \\
\hline \multicolumn{6}{|c|}{$\eta_{1}\left(q, \theta_{1}\right)$} & $=\theta_{11} e^{-\theta_{12} q^{2}}+\theta_{13} e^{-\theta_{14} q^{2}}$ \\
\hline$q_{\text {max }}^{2}$ & $n$ & $\hat{\theta}_{12}$ & $s . e$. & $v^{2}$ \\
\hline 0.010 & 579 & 135.71 & 3.0461 & 0.900 \\
0.015 & 1152 & 135.73 & 1.2808 & 1.056 \\
0.020 & 1710 & 135.21 & 1.9935 & 1.079 \\
0.025 & 2259 & 135.62 & 1.3504 & 1.093 \\
0.030 & 2808 & 135.37 & 1.1681 & 1.081 \\
0.035 & 3354 & 136.98 & 1.7161 & 1.068 \\
0.040 & 3847 & 138.59 & 1.7650 & 1.065 \\
0.097 (see text) & 5589 & 142.89 & 1.0837 & 1.115 \\
\hline
\end{tabular}

where $\phi(\theta q)=\left[\frac{\sin (\theta q)-\theta q \cos (\theta q)}{(\theta q)^{3}}\right]^{2}$. The same restrictions on the range of $q^{2}$ were used for this evaluation. For the model given in (23), the estimation procedure did not converge for the small values of $q_{\max }^{2}$ and numerical estimation problems (singular Hessian matrix) were also encountered for the remaining values of $q_{m a x}^{2}$. Therefore, this model was excluded from consideration. Table 5 , which is similar to Table 4, provides a summary of the $\theta_{12}$ estimates for the first Rayleigh-type model.

From a review of Table 5, the following conclusions/observations may be made:

- Model (22) lead to ill-conditioned least-square problems for $q_{\max }^{2} \leq 0.015$.

- The empirical $v^{2}$ is far beyond the $3 \sigma_{v}$ interval for the model (21) starting from $q_{\max }^{2}=0.020$. Model (22) has a much larger validity range and has the satisfactory $v^{2}$, except for the case with $q_{\max }^{2}=0.097$.

- The estimates of $\theta_{12}$ for the model (22) are much closer to the targeted value $(\sim 26.1 \mathrm{~nm}$, $\theta_{12}=R_{g} \sqrt{5 / 3}$.

- The standard errors of the $\theta_{12}$ estimates generally were smaller for the two-parameter model than the four-parameter model. 
Table 5: Rayleigh-type models for $\gamma=0.95,0.005<q^{2}<q_{\max }^{2}$

\begin{tabular}{|c|c|c|c|c|}
\hline \multicolumn{5}{|c|}{$\eta_{1}\left(q, \theta_{1}\right)=\theta_{11} \phi\left(\theta_{12} q\right)$} \\
\hline$q_{\text {max }}^{2}$ & $n$ & $\hat{\theta}_{12}$ & s.e. & $v^{2}$ \\
\hline 0.010 & 579 & 24.231 & 0.2325 & 0.986 \\
0.015 & 1152 & 23.685 & 0.09094 & 1.064 \\
0.020 & 1710 & 23.146 & 0.05533 & 1.112 \\
0.025 & 2259 & 22.594 & 0.04105 & 1.216 \\
0.030 & 2808 & 22.090 & 0.03406 & 1.345 \\
0.035 & 3354 & 21.684 & 0.03117 & 1.523 \\
0.040 & 3846 & 21.588 & 0.03083 & 1.661 \\
0.097 (see text) & 5589 & 21.311 & 0.02658 & 2.039 \\
\hline$\eta_{1}\left(q, \theta_{1}\right)$ & $=\theta_{11} \phi\left(\theta_{12} q\right)+\theta_{13} \phi\left(\theta_{14} q\right)$ \\
\hline$q_{\text {max }}^{2}$ & $n$ & $\hat{\theta}_{12}$ & $s . e$. & $v^{2}$ \\
\hline 0.010 & 579 & 24.182 & 0.2450 & 0.989 \\
0.015 & $*$ & $*$ & $*$ & $*$ \\
0.020 & 1710 & 28.622 & 5.9833 & 1.079 \\
0.025 & 2259 & 27.406 & 1.8274 & 1.093 \\
0.030 & 2808 & 27.953 & 0.9437 & 1.082 \\
0.035 & 3354 & 27.986 & 0.5243 & 1.068 \\
0.040 & 3846 & 27.732 & 0.3316 & 1.064 \\
0.097 (see text) & 5589 & 26.275 & 0.1212 & 1.117 \\
\hline
\end{tabular}

\section{EXPERIMENTAL DESIGN}

In the results presented in earlier sections, the longevity sampling times for the measurements with the aluminum scatterer, with the empty cell, and the cadmium shield were $t_{1}=20 \mathrm{~min}, t_{2}=$ $10 \mathrm{~min}$, and $t_{3}=45 \mathrm{~min}$, respectively. Throughout the model evaluation, the primary attention has been focused on the estimation of the $\theta_{12}$ parameter. It is known from research in the design of experiments that the precision of $\hat{\theta}$ (the estimate of $\theta$ ) depends on the times $t_{1}, t_{2}$, and $t_{3}$; i.e., for a given set of neutron beam intensities, sensitivities, and true values of the unknown parameters, the standard error of $\hat{\theta}$ may increase or decrease depending upon the choices for $t_{1}, t_{2}$, and $t_{3}$. The goal is to select those counting times so as to minimize, or at least reduce, the standard error of some or all components of the vector $\hat{\theta}$. If $\mathbf{D}(\hat{\theta})$ is a $m \times m$ covariance matrix, whose diagonal elements are the squares of the above-mentioned standard errors of the estimator $\hat{\theta}$, then it is expedient to minimize some function of this matrix [see Fedorov and Hackl (1997), Chap. 2]. For instance, we might minimize $\Psi[D(\hat{\theta})]=\sum_{\alpha=1}^{m} \mathbf{D}_{\alpha \alpha}(\hat{\theta})$. In our case, our prime interest is the estimator $\hat{\theta}_{12}$ and $\Psi[\mathbf{D}(\hat{\theta})]=\operatorname{Var}\left(\hat{\theta}_{12}\right)$. Therefore, we are looking for $\left(t_{1}, t_{2}, t_{3}\right)$, that minimize the quantity $\operatorname{Var}\left(\hat{\theta}_{12}\right)$ subject to the constraint $t_{1}+t_{2}+t_{3}=T$. Unfortunately, this optimization problem depends upon the true value of the estimated parameters (see more details in A.1). Therefore, we can construct only a locally optimal design [see Fedorov and Hackl (1997), Chap. 5.6].

We used the parameter estimates as given in Table 2 for $\gamma=0.90$ and $q^{2}>0.007$ to replace the unknown true values of $\hat{\theta}_{12}$ and assumed that $T=75 \mathrm{~min}$. It was found that the 'optimal $t_{3}$ must be equal zero. Correspondingly, the third component in our compound model (13) may be omitted. Figure 15 shows the dependence of the standard error of $\hat{\theta}_{12}$ as a function of $t_{1}$ given $t_{3}=5 \mathrm{~min}$. In this case the best $t_{1}$ is approximately $67.5 \mathrm{~min}$ and the standard error of $\hat{\theta}_{12}$ equals 0.79 . For $t_{3}=0$ the best time has aproximately the same value while the standard error of $\hat{\theta}_{12}$ equals 0.75 . 


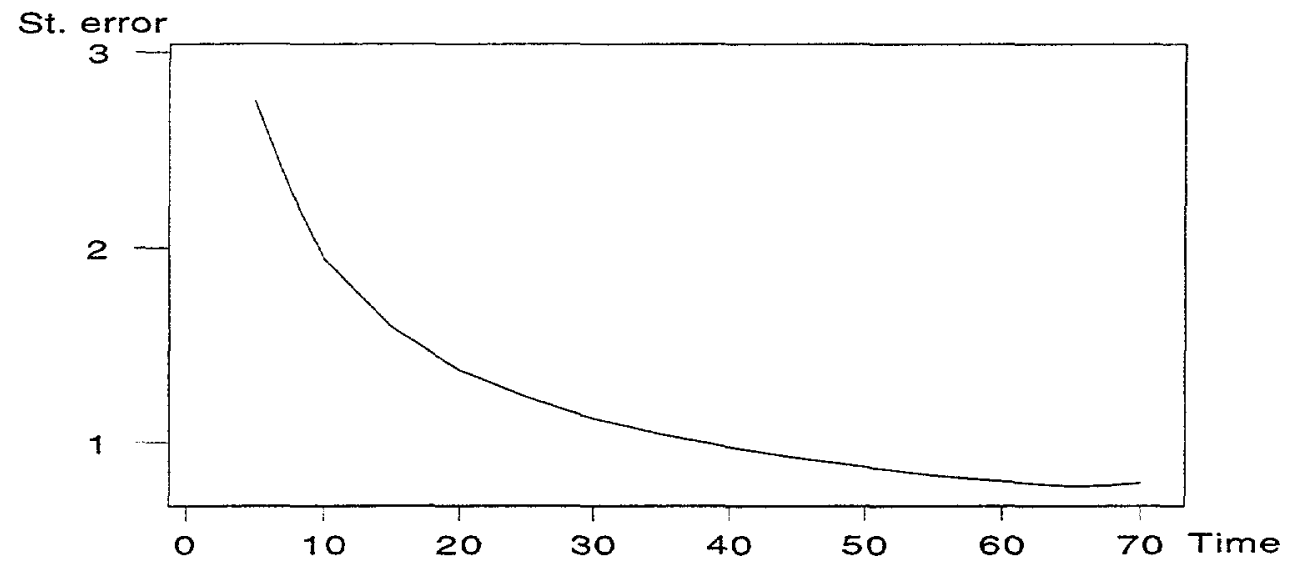

Figure 15: The standard error of $\hat{\theta}_{12}$ as a function of $t_{1}$

This value can change slightly for different ranges for $q$. Note, that for the analysed experiment we have $t_{1}=20 \mathrm{~min}, t_{2}=10 \mathrm{~min}, t_{3}=45 \mathrm{~min}$ and the standard error of $\hat{\theta}_{12}$ equals 1.38 .

The primary conclusion from this exercise is that a significant reduction in the standard error for the parameter of interest (or, equivalently, an increase in the precision) can be achieved by increasing the $t_{1}$ counting time and reducing the other counting times, if the compound model is used for parameter estimation. 


\section{APPENDIX}

\section{A.1 Iterated Maximum Likelihood Estimators}

This appendix outlines the method used to obtain the maximum likelihood estimates of the unknown parameters in the models derived in Section 2 that are used to describe the intensity observations as a function of momentum. Let $I$ be the $n \times 1$ vector of observed counts (scaled by the appropriate sensitivity as discussed in Sections 2 and 3) from the subset collection of $64 \times 64$ array of pixels to be used in the estimation phase, i.e., all restrictions on $x, y$, and $q^{2}=x^{2}+y^{2}$ have already been incorporated into the data set to eliminate any suspect detectors from consideration. If we assume the components of $I$ to be the realization of $n$ independent Poisson random variables, then the likelihood function of the sample vector $I$ is given by

$$
L(\mathbf{I}, \theta)=\prod_{i=1}^{n} \frac{\exp \left(-\mu\left(\mathbf{x}_{i}, \theta\right)\right)\left(\mu\left(\mathbf{x}_{i}, \theta\right)\right)^{I_{i}}}{I_{i} !}
$$

or

$$
l(\mathbf{I}, \theta)=\ln (L(\mathbf{I}, \theta))=\sum_{i=1}^{n} I_{i} \ln \left(\mu\left(\mathbf{x}_{i}, \theta\right)\right)-\sum_{i=1}^{n} \mu\left(\mathbf{x}_{i}, \theta\right)+\text { constant },
$$

where $\mu\left(\mathbf{x}_{i}, \theta\right)$ is the assumed model for the mean intensity similar to that used in Sections 2 and $3 ; x_{i}$ is the $k \times 1$ vector of explanatory variables associated with the $i^{\text {th }}$ pixel (e.g., $q_{x}, q_{y}, q^{2}$, or the observation times $t_{1}, t_{2}$, and $\left.t_{3}\right)$; and $\theta$ is the $m \times 1$ vector of unknown parameters to be estimated from the observed data. Our objective is to maximize $l(\mathbf{I}, \theta)$ with respect to the elements of $\theta$.

The likelihood is regular in this problem; therefore, we must find a solution of the following system of equations:

$$
\frac{\partial l(\mathbf{I}, \theta)}{\partial \theta}=\mathbf{0}
$$

where

$$
\frac{\partial l(\mathbf{I}, \theta)}{\partial \theta}=\left(\frac{\partial l(\mathbf{I}, \theta)}{\partial \theta_{1}}, \ldots, \frac{\partial l(\mathbf{I}, \theta)}{\partial \theta_{m}}\right)^{T}
$$

and 0 is a $m \times 1$ vector of zeroes. From (24) and (25) we derive that

$$
\sum_{i=1}^{n} I_{i} \frac{1}{\mu\left(\mathbf{x}_{i}, \theta\right)} \frac{\partial \mu\left(\mathbf{x}_{i}, \theta\right)}{\partial \theta}-\sum_{i=1}^{n} \frac{\partial \mu\left(\mathbf{x}_{i}, \theta\right)}{\partial \theta}=0
$$

or

$$
\sum_{i=1}^{n} w\left(\mathbf{x}_{i}, \theta\right) I_{i} \frac{\partial \mu\left(\mathbf{x}_{i}, \theta\right)}{\partial \theta}-\sum_{i=1}^{n} w\left(\mathbf{x}_{i}, \theta\right) \mu\left(\mathbf{x}_{i}, \theta\right) \frac{\partial \mu\left(\mathbf{x}_{i}, \theta\right)}{\partial \theta}=0
$$

where $w\left(\mathbf{x}_{i}, \theta\right)=1 / \mu\left(\mathbf{x}_{i}, \theta\right)$. If $\mu\left(\mathbf{x}_{i}, \theta\right)$ is expanded at the vicinity of $\theta=\theta^{(s)}$ as

$$
\mu\left(\mathbf{x}_{\mathbf{i}}, \theta^{(s)}\right)+\left(\theta-\theta^{(s)}\right)^{T} \mathbf{f}\left(\mathbf{x}_{\mathbf{i}}, \theta^{(s)}\right),
$$

where $\mathbf{f}\left(\mathbf{x}_{i}, \theta^{(s)}\right)=\partial \mu\left(\mathbf{x}_{i}, \theta^{(s)}\right) / \partial \theta$ is a $p \times 1$ vector, then, after some simplification, (26) becomes

$$
\sum_{i=1}^{n} w\left(\mathbf{x}_{i}, \theta^{(s)}\right)\left(I_{i}-\mu\left(\mathbf{x}_{i}, \theta^{(s)}\right)\right) \mathbf{f}\left(\mathbf{x}_{i}, \theta^{(s)}\right)=\sum_{i=1}^{n} w\left(\mathbf{x}_{i}, \theta^{(s)}\right) \mathbf{f}\left(\mathbf{x}_{i}, \theta^{(s)}\right) \mathbf{f}^{\mathbf{T}}\left(\mathbf{x}_{i}, \theta^{(s)}\right)\left(\theta-\theta^{(s)}\right)
$$

For any given value of $\theta^{(s)}$, the system of equations in (27) is linear in $\theta$ and may be easily solved for $\theta$. This solution takes the place of $\theta^{(s)}$, and the process is continued until convergence. Although 
convergence is not guaranteed for any choice of $\theta^{(s)}$, reasonable choices of $\theta^{(s)}$ typically lead to convergence. This method of solving for the maximum likelihood estimator of $\theta$ is referred to as iterated estimation and, in this particular setting, coincides with the iteratively reweighted least squares method and the solution is noted by $\hat{\theta}=\lim _{s \rightarrow \infty} \theta^{(s)}$ where

$$
\theta^{(s)}=\theta^{(s-1)}+\mathbf{M}^{-1}\left(\theta^{(s-1)}\right) \mathbf{Y}\left(\theta^{(s-1)}\right)
$$

$\mathbf{M}(\theta)$ is the $m \times m$ matrix

$$
\sum_{i=1}^{n} w\left(\mathbf{x}_{i}, \theta\right) \mathbf{f}\left(\mathbf{x}_{i}, \theta\right) \mathbf{f}^{T}\left(\mathbf{x}_{i}, \theta\right)
$$

and $\mathbf{Y}(\theta)$ is the $m \times 1$ vector

$$
\sum_{i=1}^{n} w\left(\mathbf{x}_{i}, \theta\right) \mathbf{f}\left(\mathbf{x}_{i}, \theta\right)\left(I_{i}-\mu\left(\mathbf{x}_{i}, \theta\right)\right)
$$

Note that $\theta^{(s)}$ in (28) is a solution of the following least-squares problem

$$
\theta^{(s)}=\arg \min _{\theta} \sum_{i=1}^{n} w\left(\mathbf{x}_{i}, \theta^{(s-1)}\right)\left[I_{i}-\mathbf{f}^{T}\left(\mathbf{x}_{i}, \theta^{(s-1)}\right) \theta^{(s-1)}\right]^{2} .
$$

As an estimate of the precision and correlation of the estimates in $\hat{\theta}$, the estimated covariance matrix may be approximated by $M^{-1}(\tilde{\theta})$, which in turn is approximately equal to

$$
M^{-1}(\hat{\theta})=\left[\sum_{i=1}^{n} w\left(\mathbf{x}_{i}, \hat{\theta}\right) \mathbf{f}\left(\mathbf{x}_{i}, \hat{\theta}\right) \mathbf{f}^{T}\left(\mathbf{x}_{i}, \hat{\theta}\right)\right]^{-1}
$$

The estimator $\hat{\theta}$ belongs to the class of, so called, iterated estimators. More detailed mathematical discussion can be found in Fedorov and Hackl (1997), Section 1.3; practical aspects are discussed in Frome et al. (1973). It is worthwhile to note that the estimator $\hat{\theta}$ is consistent and has an asymptotically normal distribution with the expectation coinciding with the true values of the estimated parameters and the covariance matrix, which can be approximated by (29).

\section{A.2 Comparison of estimators for compound and partial models}

In this section, a comparison of two approaches of estimating the parameters $\theta$ will be made. The joint method uses the maximum likelihood method to estimate the parameters in $\theta$ simultaneously using the compound model (as in (13), for instance); whereas, the second method estimates separately a subset of the components of $\theta$ and then uses these estimates to obtain estimates of the remaining components. As an example, consider data from two experiments. Let $I_{1 i}, \mathbf{x}_{1 i}$ be the collection of $n_{1}$ observations from the first experiment with

$$
E(\dot{I} \mid \mathbf{x})=\eta_{1}\left(\mathbf{x}, \theta_{1}\right)+\eta_{2}\left(\mathbf{x}, \theta_{2}\right) .
$$

Let $I_{2 i}, \mathrm{x}_{2 i}$ be the collection of $n_{2}$ observations from the second experiment with

$$
E(I \mid \mathbf{x})=\eta_{2}\left(\mathbf{x}, \theta_{2}\right)
$$

For the sake of simplicity, we assume $\operatorname{Var}(I \mid \mathbf{x})=\sigma^{2}$ for both experiments. This assumption may be easily abandoned without affecting our conclusions. In the case of a linear regression model,

$$
\eta_{1}\left(\mathbf{x}, \theta_{1}\right)=\mathbf{f}_{1}^{T}(\mathbf{x}) \theta_{1} \text { and } \eta_{2}\left(\mathbf{x}, \theta_{2}\right)=\mathbf{f}_{2}^{T}(\mathbf{x}) \theta_{2}
$$


where $\theta_{1}$ and $\theta_{2}$ are $m_{1} \times 1$ and $m_{2} \times 1$ vectors of the unknown parameters and $\mathbf{f}_{1}(\mathbf{x})$ and $\mathbf{f}_{2}(\mathbf{x})$ are $m_{1} \times 1$ and $m_{2} \times 1$ vectors, respectively, of known functions of the independent variable. It can be shown that the best linear unbiased estimator of $\theta^{T}=\left(\theta_{1}^{T} \theta_{2}^{T}\right)$ is [see Fedorov and Hackl (1997), Chap. 1]

$$
\hat{\theta}=M^{-1}\left(\begin{array}{c}
Y_{1}(1) \\
Y_{2}(1)+Y_{2}(2)
\end{array}\right)
$$

where

$$
\begin{gathered}
\mathbf{M}=\mathbf{M}(\mathbf{1})+\mathbf{M}(\mathbf{2}) \\
\mathbf{M}(\mathbf{1})=\left(\begin{array}{ll}
\mathbf{M}_{11}(1) & \mathbf{M}_{12}(1) \\
\mathbf{M}_{21}(1) & \mathbf{M}_{22}(1)
\end{array}\right) \\
\mathbf{M}(\mathbf{2})=\left(\begin{array}{cc}
0 & 0 \\
0 & \mathbf{M}_{22}(2)
\end{array}\right) \\
\mathbf{Y}_{j}(\ell)=\sum_{i=1}^{n_{1}} I_{\ell i} \mathbf{f}_{j}\left(\mathbf{x}_{i}\right)
\end{gathered}
$$

and

$$
\mathbf{M}_{\alpha \beta}(\mathbf{q})=\sum_{i=1}^{n_{q}} \mathbf{f}_{\alpha}\left(\mathbf{x}_{i}\right) \mathbf{f}_{\beta}\left(\mathbf{x}_{i}\right)
$$

for $\ell, \alpha$, and $\beta=1,2$. The estimator in (33) may also be rewritten as the following weighted sum:

$$
\hat{\theta}=W(1)\left(\begin{array}{c}
\hat{\theta}_{1}(1) \\
\hat{\theta}_{2}(2)
\end{array}\right)+W(2)\left(\begin{array}{c}
0 \\
\hat{\theta}_{2}(2)
\end{array}\right)
$$

where

$$
\mathbf{W}(\ell)=\mathbf{M}^{-1} \mathbf{M}(\ell)
$$

The partial least-squares estimators are given by

$$
\hat{\theta}(1)=\left(\begin{array}{c}
\hat{\theta_{1}}(1) \\
\hat{\theta_{2}}(1)
\end{array}\right)=\mathbf{M}^{-1}(1) \mathbf{Y}(1),
$$

and

$$
\hat{\theta}(2)=\mathbf{M}_{22}^{-1}(2) \mathrm{Y}_{2}(2)
$$

Note that

$$
\begin{gathered}
\operatorname{Var}(\hat{\theta})=\sigma^{2} \mathbf{M}^{-1}, \\
\operatorname{Var}(\hat{\theta}(\mathbf{1}))=\sigma^{2} \mathbf{M}^{-\mathbf{1}}(\mathbf{1}),
\end{gathered}
$$

and

$$
\operatorname{Var}\left(\hat{\theta}_{2}(\mathbf{2})\right)=\sigma^{2} \mathbf{M}_{22}^{-1}(2) .
$$

The interest in the partial least-squares estimators stems from the fact that in many studies the following procedure is used, especially when the parameters in $\theta_{2}$ are some auxiliary or nuisance parameters (e.g., parameters used to model the background signal or noise): 
1. Compute $\hat{\theta}_{2}(2)$ using only the data from experiment 2 .

2. Correct the data from experiment 1 by

$$
\tilde{I}_{1 i}=I_{1 i}-\hat{I}_{1 i}(2)
$$

where

$$
\hat{I}_{1 i}(2)=\mathbf{f}_{\mathbf{2}}^{T}\left(x_{i}\right) \hat{\theta}_{2}(2)
$$

3. Compute the estimate

$$
\tilde{\theta}_{1}=\mathbf{M}_{11}^{-1}(1) \tilde{Y}_{1}
$$

where

$$
\tilde{\mathbf{Y}}_{\mathbf{I}}=\sum_{i=1}^{n_{1}} \tilde{I}_{1 i} \mathbf{f}_{\mathbf{1}}\left(\mathbf{x}_{\mathbf{i}}\right)
$$

From (33), using the well-known formula of inversion of partitioned matrices [cf. Harville (1997), Chpt. 8.5],

$$
\operatorname{Var}\left(\hat{\theta_{1}}\right)=\left(\mathbf{M}^{-1}\right)_{11}=\mathrm{M}_{11}^{-1}(1)+\mathrm{M}_{11}^{-1}(1) \mathrm{M}_{12}(1) \mathbf{H}^{-1} \mathbf{M}_{21}(1) \mathbf{M}_{11}^{-1}(1)
$$

where

$$
\mathbf{H}=\mathbf{M}_{22}(1)+\mathbf{M}_{22}(2)-\mathrm{M}_{21}(1) \mathrm{M}_{11}^{-1}(1) \mathbf{M}_{12}(1) .
$$

For $\tilde{\theta_{1}}$, it can be shown that

$$
\operatorname{Var}\left(\tilde{\theta}_{1}\right)=\mathbf{M}_{11}^{-1}(1)+\mathbf{M}_{11}^{-1}(1) \mathbf{M}_{12}(1) \mathbf{M}_{22}^{-1}(2) \mathbf{M}_{21}(1) \mathbf{M}_{11}^{-1}(1)
$$

Both of the estimators are unbiased. The only difference in the expressions for the $\operatorname{Var}\left(\hat{\theta}_{1}\right)$ and $\operatorname{Var}\left(\tilde{\theta}_{1}\right)$ is the second term on the right-hand side of equations (34) and (35). Where $\mathbf{H}^{-1}$ appears in equation (34), the matrix $\mathbf{M}_{\mathbf{2 2}}^{-1}(2)$ appears in equation (35). However, it can be verified that the

$$
M_{22}(2)-M_{21}(1) M_{11}^{-1}(1) M_{12}(1)
$$

component of $\mathbf{H}$ is nonnegative definite. Therefore, in the matrix sense

$$
\mathrm{H} \geq \mathrm{M}_{22}(\mathbf{2})
$$

and consequently

$$
\operatorname{Var}\left(\hat{\theta_{1}}\right)<\operatorname{Var}\left(\tilde{\theta_{1}}\right) .
$$

Although we have considered only the linear case, the same conclusion should hold asymptotically for the nonlinear case. 


\section{REFERENCES}

Chen, S. H. and T. L. Lin (1987). "Colloidal Solutions." In Methods of Experimental Physics 23 B, Ed. D. L. Price and K. Sköld. Academic Press.

Box G.E.P. and N. Draper (1987). Empirical Model Building and Response Surfaces. New York: Wiley.

Dubner, W. S., J. M. Schulz, and G. D. Wignall (1990). "Estimation of Incoherent Backgrounds in SANS Studies of Polymers." Journal of Applied Cryst., 23, 469.

Fedorov, V. V. and P. Hackl (1997). Model-oriented Design of Experiments. New York: SpringerVerlag.

Frome, E. L., M. H. Kutner and J.J. Beauchamp (1973). "Regression Analysis of PoissonDistributed Data." Journal of the American Statistical Assotiation, 68, 935.

Hutchings, M. T. and C. G. Windsor (1987). "Industrial Applications." In Methods of Experimental Physics 23C, Ed. D. L. Price and K. Sköld. Academic Press.

Johnson, N.L. and S. Kotz (1969) Distributions in Statistics: Dicrete Distributions. Houghton Mifflin Company, Boston.

Koehler, W. (1986). "The National Center for Small-Angle Neutron Scattering." Physica, 137B, 320.

SAS Institute Inc. (1989). SAS/STAT User's Guide, Version 6, Fourth Edition, Volume 2, Cary, NC: SAS Institute Inc., 846.

Schelten J. and W. Schmatz (1980). "Multiple-Scattering Treatment for Small-Angle Scattering Problems." Journal of Applied Cryst., 13, 385.

Wignall, G. D. and F. S. Bates (1987). "Absolute Calibration of Small-Angle Neutron Scattering Data." Journal of Applied Cryst., 20, 28.

Wignall, G. D., D. K. Christen and V. Ramakrishnan (1988). "Instrumental Resolution Effects in Small-Angle Neutron Scattering." Journal of Applied Cryst., 21, 438.

Windsor, C. G. (1988). "An Introduction to Small-Angle Neutron Scattering." Journal of Applied Cryst., 21, 582 . 


\section{INTERNAL DISTRIBUTION}

1. C. K. Bayne

2-3. T. S. Darland

4-8. V. V. Fedorov

9. T. A. Gabriel

10. N. W. Grady

11-15. W. A. Hamilton
16. M. R. Leuze

17-21. M. Yethiraj

22. T. Zacharia

23. Laboratory Records - RC

24-25. Laboratory Records Dept.

26. Central Research Library

27-28. OSTI

\section{EXTERNAL DISTRIBUTION}

29. Professor A. C. Atkinson, London School of Economics, Long WC2A 2AE, United Kingdom

30-34. Professor J. J. Beauchamp, Department of Mathematics Lipscomb University Nashville, TN 37204-3651

35. Dr. R. Beckman, Statistics Group, Los Alamos National Laboratory, MS F600, Los Alamos, NM 87545

36. Professor H. Bozdogan, Department of Statistics, University of Tennessee, Knoxville, TN 37996-0532

37. Professor R. D. Cook, School of Statistics, University of Minnesota, 1994 Bufford Avenue, St. Paul, MN 55108-6042

38. Dr. R. L. A. Cottrell, MS97, SLAC, 2575 Sand Hill Road, Menlo Park, CA 94025

39. Professor D. Cox, Department of Statistics, Rice University, Houston, TX 772511892

40. G. J. Davis, Department of Mathematics, Georgia State University, Atlanta, GA 30303-3083

41. Dr. Albert Einstein C.N.R.S. - U.R.A. 1376: Bat. 4 Sophia-Antipolis Cedex France

42. Dr. Stephen T. Elbert, Division of Mathematical, Information, and Computational Sciences, Department of Energy, ER-31, 19901 Germantown Road, Germantown, MD 20874-1290

43. Professor P. Hackl, Department of Statistics, Vienna University of Economics, Augasse 2-6, A-1090 WIEN, Austria

44. Daniel A. Hitchcock, Acting Director, Division of Mathematical, Information, and Computational Sciences, Department of Energy, ER-31, 19901 Germantown Road, Room E-230, Germantown, MD 20874-1290

45. Frederick A. Howes, Division of Mathematical, Information, and Computational Sciences, Department of Energy, ER-31, 19901 Germantown Road, Room E-236, Germantown, MD 20874-1290 
46. Professor R. Mee, Department of Statistics, University of Tennessee, Knoxville, TN 37996-0532

47. Dr. W. Muller, Department of Statistics, Vienna University of Economics, Augasse 2-6, A-1090 WIEN, Austria

48. David B. Nelson, Associate Director, Office of Computational and Technology Research, Department of Energy, ER-30, 19901 Germantown Road, Room E-219, Germantown, MD 20874-1290

49. Dr. Luc Pronzato, Universite de Nice, F-06560 VALBONNE, FRANCE

50. Professor W. Welch, Department of Statistics and Actuarial Science, University of Waterloo, Waterloo, Ontario N2L, Canada

51. Professor J. Wu, Department of Statistics, Mason Hall, University of Michigan, Ann Arbor, MI 48109

52. Professor D. Ylvisaker, Department of Mathematics, University of California, Los Angeles, CA 90024 